\title{
An integrated network analysis reveals that nitric oxide reductase prevents metabolic cycling of nitric oxide by Pseudomonas aeruginosa.
}

Jonathan L. Robinson $^{\mathrm{a}, 1}$, Jacob M. Jaslove ${ }^{\mathrm{b}, \mathrm{c}}$, Allison M. Murawski ${ }^{\mathrm{b}, \mathrm{c}}$, Christopher H. Fazen ${ }^{\mathrm{a}, 2}$, and Mark P. Brynildsen ${ }^{\mathrm{a}, \mathrm{b}, *}$

a. Department of Chemical and Biological Engineering, Princeton University, Princeton, NJ 08544, USA.

b. Department of Molecular Biology, Princeton University, Princeton, NJ 08544, USA.

c. Rutgers Robert Wood Johnson Medical School, Piscataway, NJ 08854, USA.

1. Present address: Department of Biology and Biological Engineering, Chalmers University of Technology, SE412 96 Gothenburg, Sweden.

2. Present address: Department of Chemistry, The College of New Jersey, Ewing, NJ 08628, USA.

*CORRESPONDING AUTHOR:

Mark P. Brynildsen

School of Engineering and Applied Science

Department of Chemical and Biological Engineering Princeton University 205 Hoyt Laboratory 25 William Street

Princeton, NJ 08544

Phone: 609-258-1995

Fax: 609-258-0211

Email: mbrynild@princeton.edu 


\section{ABSTRACT}

Nitric oxide (NO) is a chemical weapon within the arsenal of immune cells, but is also generated endogenously by different bacteria. Pseudomonas aeruginosa are pathogens that contain an NO-generating nitrite $\left(\mathrm{NO}_{2}{ }^{-}\right)$reductase (NirS), and $\mathrm{NO}$ has been shown to influence their virulence. Interestingly, P. aeruginosa also contain NO dioxygenase (Fhp) and nitrate $\left(\mathrm{NO}_{3}{ }^{-}\right)$reductases, which together with NirS provide the potential for NO to be metabolically cycled (NO $\rightarrow \mathrm{NO}_{3}{ }^{-} \rightarrow \mathrm{NO}_{2}{ }^{-} \rightarrow \mathrm{NO}$ ). Deeper understanding of NO metabolism in $P$. aeruginosa will increase knowledge of its pathogenesis, and computational models have proven to be useful tools for the quantitative dissection of NO biochemical networks. Here we developed such a model for $P$. aeruginosa and confirmed its predictive accuracy with measurements of $\mathrm{NO}, \mathrm{O}_{2}, \mathrm{NO}_{2}{ }^{-}$, and $\mathrm{NO}_{3}{ }^{-}$in mutant cultures devoid of Fhp or NorCB (NO reductase) activity. Using the model, we assessed whether NO was metabolically cycled in aerobic $P$. aeruginosa cultures. Calculated fluxes indicated a bottleneck at $\mathrm{NO}_{3}{ }^{-}$, which was relieved upon $\mathrm{O}_{2}$ depletion. As cell growth depleted dissolved $\mathrm{O}_{2}$ levels, $\mathrm{NO}_{3}{ }^{-}$was converted to $\mathrm{NO}_{2}{ }^{-}$at near-stoichiometric levels, whereas $\mathrm{NO}_{2}{ }^{-}$consumption did not coincide with $\mathrm{NO}$ or $\mathrm{NO}_{3}{ }^{-}$accumulation. Assimilatory $\mathrm{NO}_{2}{ }^{-}$reductase (NirBD) or NorCB activity could have prevented NO cycling, and experiments with $\Delta$ nirB, $\Delta$ nirS, and $\Delta n o r C$ showed that NorCB was responsible for loss of flux from the cycle. Collectively, this work provides a computational tool to analyze NO metabolism in $P$. aeruginosa, and establishes that $P$. aeruginosa use NorCB to prevent metabolic cycling of NO.

Keywords: metabolic cycle; kinetic model; oscillations; NO reductase; Fhp; denitrification. 


\section{INTRODUCTION}

Pseudomonas aeruginosa are a common cause of infections on biomedical devices, at surgical sites, and within the airways of cystic fibrosis patients [1,2]. Treatment of $P$. aeruginosa infections is difficult because they are Gram-negative bacteria that are avid biofilm formers $[3,4]$ and they possess both broad-spectrum $\beta$-lactamases and numerous multidrug efflux pumps, which make them naturally resistant to many antibiotics $[5,6]$. Further, antibiotics that were once effective against $P$. aeruginosa, such as fluoroquinolones and aminoglycosides, are now innocuous to many clinical isolates due to the rise and spread of multidrug resistant (MDR) strains [7]. This confluence of challenges has motivated many searches for new agents or methodologies to treat $P$. aeruginosa infections [8-11]. One particularly promising avenue involves targeting the processes that pathogens use to cause illness rather than those that are essential to its propagation under any circumstance [11]. Conventional antibiotics fall into the latter category, applying selective pressure both inside and outside the human body (e.g., sewage, soil, water), whereas antivirulence therapies comprise the former category, confining selective pressure to within the host [12-15]. Such agents would be orthogonal to current treatments, and their focused nature is projected to produce resistance at far greater time scales than antibiotics [12].

To facilitate the development of antivirulence therapies, much work has been conducted to increase understanding of $P$. aeruginosa pathogenesis and its physiology within host environments [6,16-18]. During pathogenesis, $P$. aeruginosa are exposed to nitric oxide (NO) generated by immune cells, such as macrophages $[19,20]$. NO is a toxic metabolite that readily reacts with iron-sulfur clusters ([Fe-S]), heme cofactors, and other transition metal centers, and several of its autoxidation products can damage free or protein-bound thiols, tyrosine residues, 
and DNA bases [21-24]. To counteract the nitrosative assault of immune cells, $P$. aeruginosa employ detoxification systems, such NO reductase and NO dioxygenase, which are encoded by nor $C B$ and $f h p$, respectively [25,26]. In essence, $P$. aeruginosa employs these enzymes to divert flux of NO away from reactions that damage the pathogen (e.g., nitrosylation of Fe-S clusters) toward reactions that neutralize this toxic metabolite (e.g., reduction to $\mathrm{N}_{2} \mathrm{O}$ ). From a metabolic engineering perspective, this is analogous to a biocatalyst (P. aeruginosa) that has metabolic pathways (NO detoxification) that divert substrate (NO) away from the desired pathway (damage to biomolecules), and thus reduce product yield (killing of P. aeruginosa) [27]. A strategy to improve desirable outcomes would be to eliminate competing pathways, and Kakishima and colleagues have shown this with $P$. aeruginosa and NO detoxification where $P$. aeruginosa devoid of NorCB were killed more quickly than wild-type (WT) by LPS-activated macrophages, and that the survival difference was eliminated with use of an inducible nitric oxide synthase (iNOS) inhibitor [20].

Beyond improving survival in macrophages, $P$. aeruginosa NO metabolism has been associated with numerous pathogenic processes [28-31]. For example, NO was found to stimulate dispersal of $P$. aeruginosa biofilms by increasing phosphodiesterase activity, which reduced the level of cyclic-di-GMP [29]. Endogenous NO production by $P$. aeruginosa from one of its $\mathrm{NO}_{2}^{-}$reductases (NirS) has been identified as a mediator of both virulence factor expression and biofilm dispersal [28,31]. Van Alst and colleagues observed that both $\Delta n a r G H$ and $\Delta$ nirS P. aeruginosa were far less virulent to Caenorhabditis elegans than the WT [31]. In a later study, the same mutants were shown to be more sensitive to killing by human monocytes, and found to be defective in expression of a type III secretion system [30]. Barraud and colleagues observed that biofilms of $\Delta$ nirS dispersed far less than those of WT, and provided 
evidence that low concentrations of $\mathrm{NO}$ were the cause [28]. Importantly, $\mathrm{NO}_{2}{ }^{-}$is a relatively stable end product of the reaction of $\mathrm{NO}$ with $\mathrm{O}_{2}$, and can therefore be present at infection sites [32].

The influence of the $P$. aeruginosa NO network on pathogenic processes suggests that deeper quantitative understanding of how it functions as a system could reveal strategies to sabotage $P$. aeruginosa pathogenesis. In this regard, a parallel can be drawn to metabolic engineering, where deeper knowledge of how metabolism functions as an interconnected system will reveal ways to improve chemical production. Such knowledge is provided with the use of computational models [33-35], and the same applies to NO, which is a highly reactive molecule with an expansive biochemical reaction network [24,36-38]. Computational models of NO biochemistry and regulation have increased understanding of NO biology from mammalian to bacterial systems [36-42], and in one recent example, we used a model of E. coli NO stress to unravel the mechanism behind $\mathrm{NO}$ oscillations in low $\mathrm{O}_{2}$ environments [43]. With respect to $P$. aeruginosa and NO, a previous mathematical model of the regulatory network associated with denitrification was developed by Arat and colleagues [44]. In that study, a qualitative, discretevariable model of the $P$. aeruginosa denitrification regulatory network was constructed to understand the impact of environmental variables, such as phosphate and $\mathrm{O}_{2}$, on the system [44]. Notably, that model was time-independent, encompassed only the denitrification pathway $\left(\mathrm{NO}_{3}{ }^{-}\right.$ to $\mathrm{N}_{2}$ ), and was qualitative in the sense that all variables were either Boolean or ternary ("low", “medium", "high"). In addition, a genome-scale metabolic model of P. aeruginosa has been constructed $[45,46]$, which provides a valuable resource and framework for studies of the $P$. aeruginosa metabolic network [47-49]; however, the absence of kinetics and many NO-specific reactions precludes its use for dynamic analyses of NO stress in P. aeruginosa. 
In this work, we had two goals: (1) develop a quantitative model of the $P$. aeruginosa NO network that would provide a computational tool for examination of the dynamics of this metabolic system, and (2) assess whether an NO cycle present in the metabolic network was functional (Fig. 1). Specifically, P. aeruginosa contains enzymes that can perform the conversion of $\mathrm{NO} \rightarrow \mathrm{NO}_{3}{ }^{-} \rightarrow \mathrm{NO}_{2}^{-} \rightarrow \mathrm{NO}$. This cycle is composed of NO dioxygenase, $\mathrm{NO}_{3}{ }^{-}$reductase, and $\mathrm{NO}_{2}{ }^{-}$reductase activities, which would collectively deactivate $\mathrm{NO}$, only to regenerate it in a subsequent enzymatic step. Interestingly, elevated levels of $\mathrm{NO}_{2}{ }^{-}$and $\mathrm{NO}_{3}{ }^{-}$have been found in the lungs of cystic fibrosis patients, who often suffer from P. aeruginosa infections, and this has been interpreted as an indication of increased NO production by host immune cells, which suggests that $P$. aeruginosa infection environments contain all three metabolites of the NO cycle $[32,50]$. In addition, the $P$. aeruginosa $\mathrm{NO}$ cycle network architecture is distinct from that of Escherichia coli, which has served as a model Gram-negative bacterium for the study of NO metabolism [21,36,51,52], because the $\mathrm{NO}_{2}{ }^{-}$reductases of $E$. coli generate ammonia $\left(\mathrm{NH}_{3}\right)$ rather than NO [53,54], and thus $E$. coli does not have the capacity to cycle NO. In fact, $P$. aeruginosa has a similar, additional $\mathrm{NO}_{2}{ }^{-}$reductase (NirBD) that produces $\mathrm{NH}_{3}$, which would prevent an $\mathrm{NO}$ cycle, as well as an $\mathrm{NO}$ reductase (NorCB) that would convert $\mathrm{NO}$ to $\mathrm{N}_{2} \mathrm{O}$ $[26,55,56]$. Due to these potential exit strategies and differences that exist in the regulation of these enzymes [55,57], it was uncertain whether an NO cycle would occur.

A

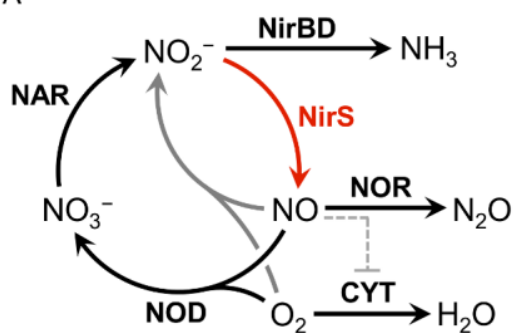

B

\begin{tabular}{|c|c|c|}
\hline Function & Enzyme & Reaction \\
\hline NOD & Fhp & $2 \mathrm{NO}+2 \mathrm{O}_{2}+2 \mathrm{NADH} \rightarrow 2 \mathrm{NO}_{3}{ }^{-}+\mathrm{NAD}^{+}+\mathrm{H}^{+}$ \\
\hline \multirow{2}{*}{ NAR } & $\mathrm{NarGHI}$ & cytoplasm: $\mathrm{NO}_{3}{ }^{-}+\mathrm{Q}_{8} \mathrm{H}_{2} \rightarrow \mathrm{NO}_{2}{ }^{-}+\mathrm{Q}_{8}+\mathrm{H}_{2} \mathrm{O}$ \\
\cline { 2 - 3 } & $\mathrm{NapAB}$ & periplasm: $\mathrm{NO}_{3}{ }^{-}+\mathrm{Q}_{8} \mathrm{H}_{2} \rightarrow \mathrm{NO}_{2}{ }^{-}+\mathrm{Q}_{8}+\mathrm{H}_{2} \mathrm{O}$ \\
\hline \multirow{2}{*}{ NIR } & NirBD & $\mathrm{NO}_{2}{ }^{-}+3 \mathrm{NADH}+4 \mathrm{H}^{+} \rightarrow \mathrm{NH}_{3}+3 \mathrm{NAD}^{+}+2 \mathrm{H}_{2} \mathrm{O}$ \\
\cline { 2 - 3 } & NirS & $\mathrm{NO}_{2}{ }^{-}+\mathrm{cytc}_{\mathrm{Fe}^{2+}}+2 \mathrm{H}^{+} \rightarrow \mathrm{NO}+\mathrm{cytc}_{\mathrm{Fe}^{3+}}+\mathrm{H}_{2} \mathrm{O}$ \\
\hline NOR & NorCB & $2 \mathrm{NO}+\mathrm{Q}_{8} \mathrm{H}_{2} \rightarrow \mathrm{N}_{2} \mathrm{O}+\mathrm{H}_{2} \mathrm{O}+\mathrm{Q}_{8}$ \\
\hline
\end{tabular}

Figure 1. NO metabolic cycle subnetwork. (A) Simplified reaction network diagram illustrating the $\mathrm{NO} \rightarrow \mathrm{NO}_{3}{ }^{-} \rightarrow \mathrm{NO}_{2}{ }^{-} \rightarrow \mathrm{NO}$ metabolic cycle present in $P$. aeruginosa. Relevant reactions that remove flux from the cycle (catalyzed by NirBD and NOR) are also shown, as well as the NO-inhibited respiratory cytochromes (CYT) 
that compete with NOD for available $\mathrm{O}_{2}$. Enzymes are in bold, near the reaction they catalyze. The autoxidation of $\mathrm{NO}$ by $\mathrm{O}_{2}$ (gray arrow) is spontaneous, and thus has no associated enzyme. The network is comparable to that of $E$. coli, except $E$. coli lack an NO-generating $\mathrm{NO}_{2}{ }^{-}$reductase (shown in red), thus preventing the metabolic cycling of NO. For illustrative purposes, the reactions were not balanced in element or charge; thus, the table in (B) provides the balanced chemical equations. $\mathrm{Q}_{8} \mathrm{H}_{2}$ is ubiquinol; $\mathrm{Q}_{8}$ is ubiquinone; cyt $c_{\mathrm{Fe} 2+}$ and $\mathrm{cyt} c_{\mathrm{Fe}+}$ are the ferrous and ferric forms of cytochrome $c$, respectively.

To accomplish the first of our goals, we constructed a quantitative, kinetic model of NO stress in P. aeruginosa, and experimentally validated its predictions of NO metabolism in mutants devoid of NO dioxygenase and NO reductase, $\Delta f h p$ and $\Delta n o r C$, respectively. This model can serve as a valuable tool to calculate fluxes of NO through cellular and non-cellular pathways in $P$. aeruginosa cultures, which is an important task for any metabolic system where competition for substrate limits desirable outcomes. Using this model, we calculated fluxes through $\mathrm{NO}$ dioxygenase, $\mathrm{NO}_{3}{ }^{-}$reductase, and $\mathrm{NO}_{2}{ }^{-}$reductase during and immediately following $\mathrm{NO}$ stress, which suggested that $\mathrm{NO}_{3}{ }^{-}$reductase was not functioning appreciably. Given the role of $\mathrm{O}_{2}$ in regulation of $\mathrm{NO}_{3}{ }^{-}$reductase activity $[58,59]$, we allowed cultures to grow and deplete $\mathrm{O}_{2}$ from the media. When dissolved $\mathrm{O}_{2}$ concentrations dropped to $\sim 3 \mu \mathrm{M}$, $\mathrm{NO}_{3}{ }^{-}$consumption and $\mathrm{NO}_{2}{ }^{-}$accumulation were observed. Interestingly, $\mathrm{NO}$ became briefly detectable upon $\mathrm{NO}_{2}{ }^{-}$accumulation, but the quantitative amounts were small and $\mathrm{NO}_{3}{ }^{-}$failed to accumulate again, which pointed to a mechanism by which $P$. aeruginosa prevented the NO cycle. Subsequent analyses with genetic mutants identified NO reductase (NorCB) as the major enzyme removing flux from the NO cycle, and in its absence, NO concentrations oscillated with an amplitude (defined here as the peak NO concentration) of $\sim 3 \mu \mathrm{M}$ and period of $\sim 3$ minutes. These oscillations were consistent with a recently identified stress-metabolic oscillator that originates from a competition for $\mathrm{O}_{2}$ between $\mathrm{NO}$ dioxygenase and aerobic respiratory terminal oxidases [43]. Given the difference in oscillation dynamics from those observed previously, we evaluated the impact of varying key model parameters on oscillation behavior. The analysis 
revealed that the oscillation period and amplitude are sensitive to many changes in species and culturing conditions, and identified NO-mediated substrate inhibition of NO dioxygenase as a necessary feature for oscillations. Finally, to provide additional experimental support for the NO cycling mechanism and the associated role of NorCB, we demonstrated that the NO cycle can be initiated by the addition of $\mathrm{NO}_{3}{ }^{-}$to $\triangle$ norC cultures, but that it is not appreciably active when NorC is present. Collectively, this study provides a computational tool to quantitatively analyze NO fluxes through desired and competing reactions in $P$. aeruginosa cultures, and demonstrates that NorCB prevents cycling of NO in P. aeruginosa cultures.

\section{RESULTS}

\subsection{Construction of a quantitative kinetic model of NO metabolism in P. aeruginosa}

\subsubsection{Model construction}

A quantitative kinetic model of the NO stress network in P. aeruginosa PAO1 was constructed using an existing $E$. coli K-12 MG1655 model [43] as a template, and following a procedure that was recently implemented to develop a model for enterohemorrhagic E. coli O157:H7 [60] (see Section 4.7 for additional construction details, Table S1 for model species, Tables S2 and S3 for model reactions, and Model S1 for the complete model file). A large amount of the $E$. coli model structure was retained, including 113 biochemical species (77\% of the $E$. coli model), 119 reaction rate expressions (69\%), and 131 kinetic parameters (69\%), but there were also a number of substantial changes needed to adapt the model to $P$. aeruginosa. The most notable differences between the $E$. coli and $P$. aeruginosa networks involved the aerobic respiratory quinol oxidases and the denitrification enzymes. E. coli possesses three aerobic $\left(\mathrm{O}_{2}-\right.$ consuming) cytochrome quinol oxidases: cytochromes bo, bd-I, and bd-II (Cyo, Cyd, and 
AppCB, respectively) [53]. P. aeruginosa, however, harbors five different cytochrome terminal oxidases, two of which are ubiquinol oxidases (Cyo and cyanide insensitive oxidase (CIO)), whereas the other three are cytochrome $c$ oxidases (Cbb3-1, Cbb3-2, and Aa3) [61,62]. The three E. coli quinol oxidases in the model were therefore replaced with the five $P$. aeruginosa systems. The $\mathrm{NO}, \mathrm{NO}_{2}{ }^{-}$, and $\mathrm{NO}_{3}{ }^{-}$processing pathways share some similarities between $E$. coli and $P$. aeruginosa; specifically, NO dioxygenase (Hmp in E. coli, Fhp in $P$. aeruginosa), and $\mathrm{NO}, \mathrm{NO}_{2}{ }^{-}$, and $\mathrm{NO}_{3}{ }^{-}$reductases (NOR, NIR, and NAR, respectively) are present in both species. However, $P$. aeruginosa possesses an additional type of NIR that reduces $\mathrm{NO}_{2}^{-}$to generate $\mathrm{NO}$ (dissimilatory NIR, NirS) in addition to a NIR that generates $\mathrm{NH}_{3}$ (assimilatory NIR, NirBD). This is of particular interest because NirS would enable NO to be generated endogenously from $\mathrm{NO}_{2}{ }^{-}$(Fig. 1), which is a function that has been shown previously to modulate biofilm dispersal and expression of virulence factors in $P$. aeruginosa $[28,29]$. Therefore, the NIR and NAR enzymes of $P$. aeruginosa were added to the model. Furthermore, the transcriptional regulatory network governing expression of these and the NO detoxification enzymes was reconstructed in the PAO1 model to account for differences in regulatory structure from that of $E$. coli. This involved the addition of three transcription factors to the PAO1 model: the anaerobic response regulator (ANR), denitrification response regulator (DNR), and a two-component $\mathrm{NO}_{3}{ }^{-}$sensing regulator (NarXL) (Section 4.7 and Fig. S1) [61]. These three transcription factors and their regulatory interactions were also included in the $P$. aeruginosa regulatory network constructed by Arat and colleagues [44]; however, the present implementation enables quantitative analysis of time-dependent changes in this pathway, and does so in the context of a larger, more expansive network. 


\subsubsection{Model training}

After model construction was completed, the uncertain or unavailable parameters were trained on experimental measurements. The extracellular (organism-independent) parameters governing processes specific to the experimental apparatus were optimized first, and those were the rate of NO exchange with the gas phase, dissociation rate of DPTA NONOate to release NO, and the rate of spontaneous NO autoxidation in the media (Table S4). The parameters were optimized to simultaneously minimize error (sum of the squared residuals, SSR) between simulated and experimentally measured $\mathrm{NO}, \mathrm{NO}_{2}{ }^{-}$, and $\mathrm{NO}_{3}{ }^{-}$concentrations following treatment of cell-free growth media with $50 \mu \mathrm{M}$ DPTA (Section 4.8.1). Although the [NO] was measured continuously (> $1 \mathrm{read} / \mathrm{sec})$, only the data points corresponding to the 5 times at which $\left[\mathrm{NO}_{2}{ }^{-}\right]$ and $\left[\mathrm{NO}_{3}{ }^{-}\right]$were measured $(0,0.5,1.0,1.5$, and $2.0 \mathrm{~h}$ post-DPTA dose $)$ were used, to avoid unevenly weighting the optimization toward [NO] data. Once the optimized parameter values were obtained, a subsequent MCMC procedure was performed whereby a random walk through parameter space was conducted to identify additional sets of parameter values that were sufficiently similar in agreement with the data (evidence ratio, ER $<10$ ), yielding an ensemble of viable models (Section 4.8.2) $[43,63]$. The resulting $[\mathrm{NO}],\left[\mathrm{NO}_{2}{ }^{-}\right]$, and $\left[\mathrm{NO}_{3}{ }^{-}\right]$curves simulated by the ensemble were in excellent agreement with experimental measurements (Fig. S2).

Optimization of cellular parameters was performed in modules to improve computational feasibility by reducing the available solution space (Section 4.8.3). The respiratory module was trained first, where parameters governing aerobic respiration, 27 in total, were optimized to fit $\left[\mathrm{O}_{2}\right]$ data measured in untreated PAO1 cultures (Table S5). The optimization process mirrored that described for the extracellular parameters, where a subsequent MCMC procedure was performed to allow estimation of parameter and prediction uncertainty. The simulated $\left[\mathrm{O}_{2}\right]$ curve 
generated using the ensemble of models exhibited excellent agreement with experimental measurements (Fig. S3).

The final model training process involved all the remaining cellular parameters governing NO-stress related damage, inhibition, detoxification, and repair. A total of 137 parameters were optimized to simultaneously fit $[\mathrm{NO}]$ and $\left[\mathrm{O}_{2}\right]$ curves, as well as $\left[\mathrm{NO}_{2}{ }^{-}\right]$and $\left[\mathrm{NO}_{3}{ }^{-}\right]$at $0.5 \mathrm{~h}$ post-DPTA treatment, measured in a WT culture of $P$. aeruginosa treated with $50 \mu \mathrm{M}$ DPTA (Table S6). To incorporate uncertainty of cell-free and respiratory parameter values into the final ensemble of models, those parameters were included with the other 137 cellular parameters in the subsequent MCMC analysis and permitted to vary within their confidence intervals (CIs), which were determined in the above-mentioned steps (Section 4.8.3). Simulations run using the resulting ensemble were in good agreement with measured $[\mathrm{NO}],\left[\mathrm{O}_{2}\right],\left[\mathrm{NO}_{2}{ }^{-}\right]$, and $\left[\mathrm{NO}_{3}{ }^{-}\right]$data (Fig. 2A).
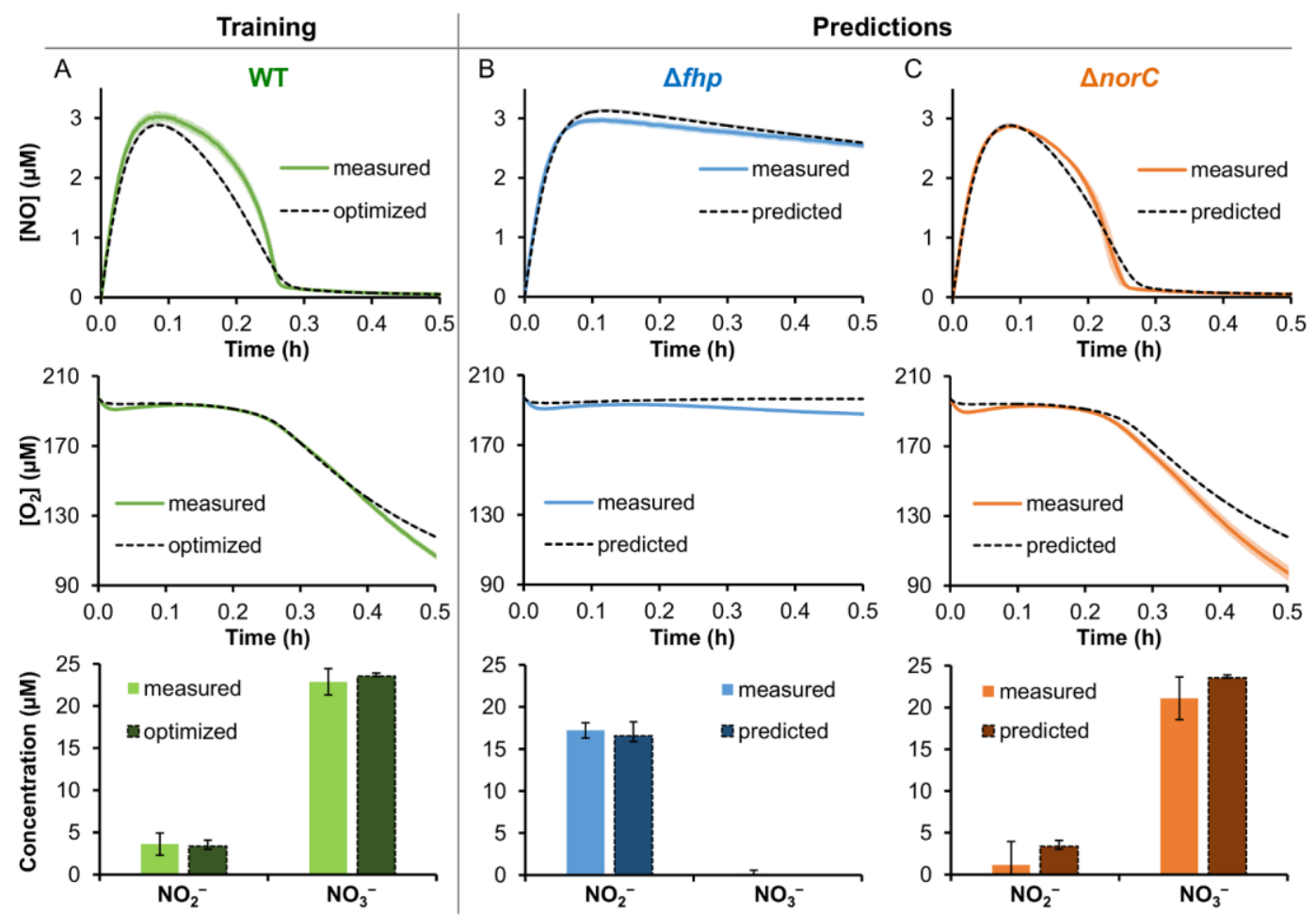

Figure 2. Model training and experimental validation. (A) The model was trained on continuous measurements of $[\mathrm{NO}]$ and $\left[\mathrm{O}_{2}\right]$, and a single measurement at $0.5 \mathrm{~h}$ of $\left[\mathrm{NO}_{2}{ }^{-}\right]$and $\left[\mathrm{NO}_{3}{ }^{-}\right]$in aerobic wild-type PAO1 cultures at an initial $\mathrm{OD}_{600}$ of 0.05 , treated with $50 \mu \mathrm{M}$ DPTA at time zero. The ensemble of viable models was used to predict 
[NO], $\left[\mathrm{O}_{2}\right],\left[\mathrm{NO}_{2}{ }^{-}\right]$, and $\left[\mathrm{NO}_{3}{ }^{-}\right]$in cultures of PAO1 mutants (B) $\Delta f h p$ and (C) $\Delta n o r C$, and the corresponding experiments were conducted and are shown. All measurements (solid lines or columns) are the mean of 3 independent experiments, with light shading (or error bars) representing the standard error of the mean (SEM). Dashed lines (or dashed-border columns) are simulation results obtained using the best-fit optimized parameter set, with gray shading (or error bars) representing parametric variation among the ensemble of viable $(\mathrm{ER}<10)$ models.

\subsubsection{Experimental validation of model predictions}

The predictive accuracy of the model was assessed by comparing simulated and measured $[\mathrm{NO}],\left[\mathrm{O}_{2}\right],\left[\mathrm{NO}_{2}{ }^{-}\right]$, and $\left[\mathrm{NO}_{3}{ }^{-}\right]$data for cultures of $P$. aeruginosa mutants lacking either of the two major NO detoxification systems: NO dioxygenase $(\Delta f h p)$ and NO reductase ( $\triangle$ nor $C$ ). Simulations predicted negligible differences in $\mathrm{NO}, \mathrm{O}_{2}, \mathrm{NO}_{2}{ }^{-}$, and $\mathrm{NO}_{3}{ }^{-}$concentrations between WT and $\Delta n o r C$ cultures, whereas the $\Delta f h p$ mutant was predicted to exhibit severe inhibition of NO detoxification and, as a result, respiration. The greater importance of Fhp compared to NorCB under these conditions was expected, given that $\mathrm{NO}$ dioxygenases have been shown to dominate NO detoxification under aerobic conditions, whereas anaerobic conditions are favorable for NO reductase activity [25,26,36,43,64,65]. The corresponding experiments were performed, for which cultures of $\Delta f h p$ and $\Delta n o r C$ were treated with $50 \mu \mathrm{M}$ DPTA NONOate under aerobic conditions. The predicted and measured $[\mathrm{NO}]$ and $\left[\mathrm{O}_{2}\right]$ curves, as well as $\left[\mathrm{NO}_{2}{ }^{-}\right]$and $\left[\mathrm{NO}_{3}{ }^{-}\right]$at $0.5 \mathrm{~h}$ post-dose were in excellent agreement for both mutants, demonstrating that the model had correctly predicted the contributions of Fhp and NorCB to aerobic NO detoxification (Figs. 2 B and C, respectively).

\subsection{Computational analysis of NO cycle activity}

An interesting difference between the NO biochemical reaction networks of E. coli and $P$. aeruginosa is the presence of a NO-generating $\mathrm{NO}_{2}^{-}$reductase in $P$. aeruginosa $(\mathrm{NirS})$. With NirS, $P$. aeruginosa could conceivably detoxify $\mathrm{NO}$ with Fhp to form $\mathrm{NO}_{3}{ }^{-}$, the $\mathrm{NO}_{3}{ }^{-}$could be 
processed by NAR to generate $\mathrm{NO}_{2}^{-}$, and NirS could generate $\mathrm{NO}$ from the resulting $\mathrm{NO}_{2}{ }^{-}$(Fig. 1). This three-enzyme system forms a biochemical equivalent to "kicking the can down the road", where NO would be chemically pacified only to arise again at a future time. Such a scenario could be prevented if $\mathrm{NO}_{2}^{-}$were processed by the assimilatory reductase, NirBD, which yields $\mathrm{NH}_{3}$ rather than $\mathrm{NO}$, or if $\mathrm{NO}$ were detoxified by $\mathrm{NO}$ reductase, NorCB, which yields $\mathrm{N}_{2} \mathrm{O}$ rather than $\mathrm{NO}_{3}{ }^{-}$(Fig. 1). Given the data presented in Fig. 2, which demonstrated that Fhp was a major cellular consumption pathway under the conditions investigated here, we sought to assess whether aerobic $P$. aeruginosa cultures execute this NO cycle. To do this, we calculated the flux through each of the enzymes comprising the NO metabolic cycle, as well as the distribution of NO flux through all other available pathways, using the ensemble of viable models that we had constructed.

Treatment of WT PAO1 with $50 \mu \mathrm{M}$ DPTA NONOate was simulated by the ensemble and the results were consistent with the large impact of $\Delta f h p$ on $\mathrm{NO}$ detoxification dynamics (Fig. 3A); Fhp was predicted to account for the majority of NO consumption, reaching $\sim 24 \mu \mathrm{M}$ NO by $0.5 \mathrm{~h}$, compared to the $\sim 5 \mu \mathrm{M}$ NO consumed by autoxidation in the media and loss to the gas phase. Flux of NO through other pathways, such as [Fe-S] nitrosylation, was negligible, cumulatively accounting for less than $0.03 \mu \mathrm{M}$ of NO consumed. Deletion of fhp from the ensemble eliminated the majority of cellular-related NO consumption, leaving autoxidation and escape to the gas phase as the two major pathways (Fig. 3B), though we note that flux through reactions that damage biomolecules were generally increased (e.g., $[\mathrm{Fe}-\mathrm{S}]$ nitrosylation increased to a maximum of $0.09 \mu \mathrm{M}$ cumulative $\mathrm{NO}$ consumed). Removal of nor $C$ exhibited nearly the same metabolic flux distribution as WT (Fig. 3C). 

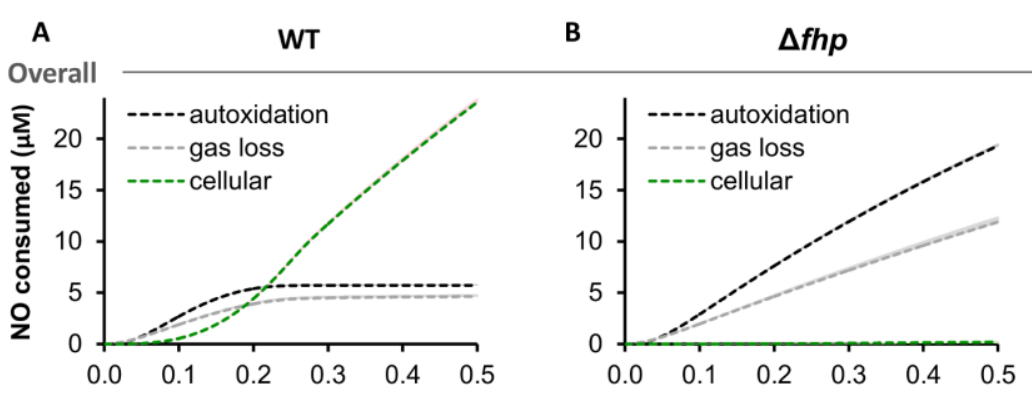

C $\quad \Delta$ norC
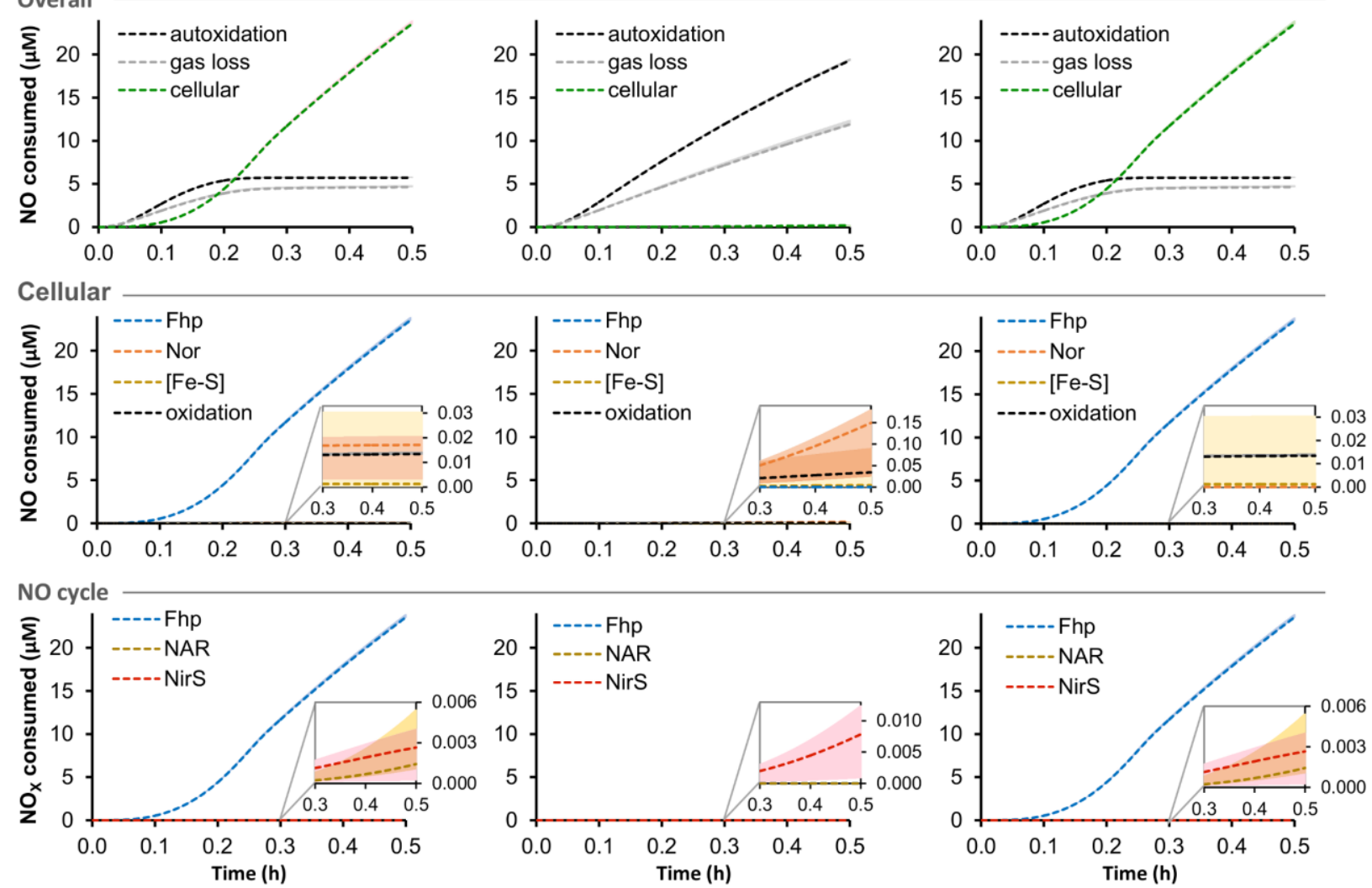

Figure 3. Simulated flux through the NO biochemical network of $P$. aeruginosa. Using the ensemble of models, DPTA treatment ( $50 \mu \mathrm{M})$ of (A) WT, (B) $\Delta f h p$, and (C) $\Delta n o r C$ PAO1 cultures was simulated, and the cumulative consumptions of NO by the major overall pathways (autoxidation in the media, loss to the gas phase, and cellular consumption) and the intracellular pathways (dioxygenation by Fhp, reduction by NorCB, [Fe-S] nitrosylation, and "oxidation," which includes the reaction of $\mathrm{NO}$ with $\mathrm{O}_{2}$, superoxide, and hydroxyl radicals) were determined. In addition, the cumulative consumptions of $\mathrm{NO}, \mathrm{NO}_{2}^{-}$, and $\mathrm{NO}_{3}^{-}$(" $\mathrm{NO}_{\mathrm{X}}$ ") through the three enzymes participating in the NO metabolic cycle (Fhp, NAR, and NirS) were calculated. Dashed lines are simulation results obtained using the best-fit model, with light shading of the same color representing the range of predictions among ensemble members. The insets are a zoomed-in region to show consumption values that are otherwise too small to view.

In terms of the NO cycle, the calculated $\mathrm{NO}_{\mathrm{X}}$ consumption by NAR and NirS in WT were orders of magnitude lower than that of Fhp, reaching a cumulative consumption (by $0.5 \mathrm{~h}$ post-dose) of only $0.001-0.005 \mu \mathrm{M} \mathrm{NO}_{3}{ }^{-}$and $0.0002-0.004 \mu \mathrm{M} \mathrm{NO}_{2}{ }^{-}$, respectively. Similar results were observed when simulating $\Delta f h p$ and $\Delta n o r C$ mutants, except for the complete elimination of NAR activity in $\Delta f h p$ due to the absence of $\mathrm{NO}_{3}{ }^{-}$supply by Fhp. These predictions suggested that the full cycle (NO $\rightarrow \mathrm{NO}_{3}{ }^{-} \rightarrow \mathrm{NO}_{2}{ }^{-} \rightarrow \mathrm{NO}$ ) was not active under the conditions used here, and that it primarily stalled at $\mathrm{NO}_{3}{ }^{-}$. The low activities of the $\mathrm{NO}_{3}{ }^{-}$and $\mathrm{NO}_{2}{ }^{-}$reductases were likely a 
result of the aerobic environment, as these enzymes function optimally under anaerobic to microaerobic conditions $[59,61]$. We therefore reasoned that progression through the NO cycle might occur as $\mathrm{O}_{2}$ depleted as a result of respiration and cell growth.

\subsection{Quantification of $\mathrm{NO}, \mathrm{NO}_{3}{ }^{-}$, and $\mathrm{NO}_{2}{ }^{-}$dynamics in WT $P$. aeruginosa cultures}

With the hypothesis that cell-mediated depletion of $\mathrm{O}_{2}$ from the culture could activate the other branches of the NO cycle, we extended the duration of experiments. Following relief of NO-induced respiratory inhibition, which occurred at $\sim 0.25 \mathrm{~h}$ post DPTA treatment, $\mathrm{O}_{2}$ was consumed rapidly and periodic quantification of $\left[\mathrm{NO}_{2}^{-}\right]$and $\left[\mathrm{NO}_{3}{ }^{-}\right]$revealed that virtually all $\mathrm{NO}_{2}{ }^{-}$and $\mathrm{NO}_{3}{ }^{-}$generated from DPTA was consumed by $3.5 \mathrm{~h}$ post-treatment (Fig. 4A).
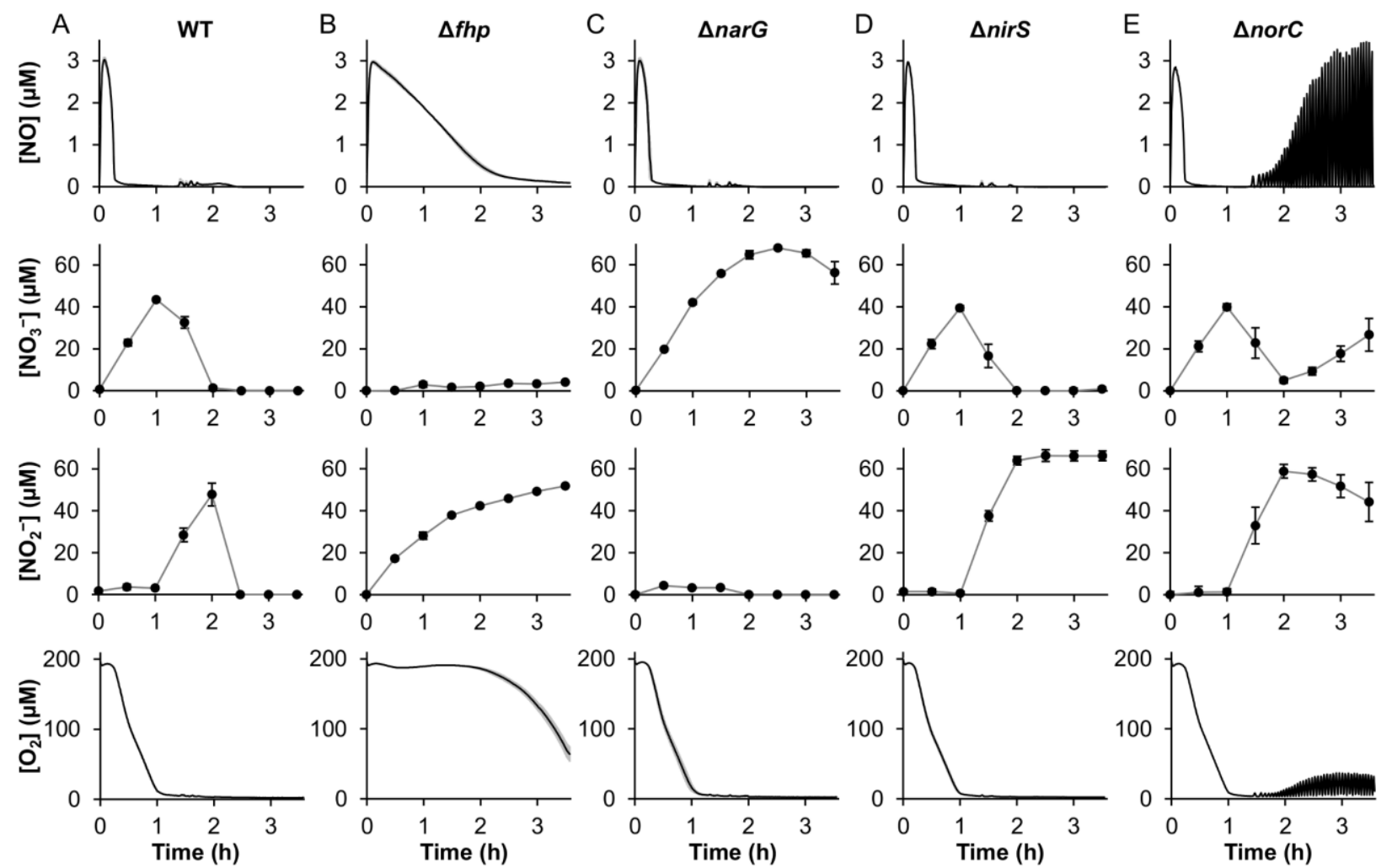

Figure 4. Genetic analysis of NO metabolic cycle following DPTA treatment. (A) WT, (B) $\Delta f h p$, (C) $\Delta n a r G$, (D) $\Delta n i r S$, and (E) $\Delta n o r C$ PAO1 cultures at an initial $\mathrm{OD}_{600}$ of 0.05 were treated with $50 \mu \mathrm{M}$ DPTA at time zero, and monitored for $3.5 \mathrm{~h}$ post-dose. Concentrations of $\mathrm{NO}$ and $\mathrm{O}_{2}$ were measured continuously, whereas $\mathrm{NO}_{2}{ }^{-}$and $\mathrm{NO}_{3}{ }^{-}$ were quantified every $0.5 \mathrm{~h}$. Solid black lines (or circles) are the mean of 3 independent experiments, with gray shading (or error bars) representing the SEM. The exception is [NO] and $\left[\mathrm{O}_{2}\right]$ of $\Delta n o r C$, which are data from one 
representative experiment because averaging multiple experiments obscures the oscillatory dynamics (see Fig. S11 for remaining $\triangle$ norC data).

We note that the computational model constructed in the previous sections was not designed to account for the complexities associated with transitioning from an antimicrobial-induced bacteriostasis to an unstressed growing state. Therefore, computational analyses were not used to examine experimental data beyond $0.5 \mathrm{~h}$, which was the final measured time point at which the cell density of the culture was not statistically higher than that of the initial time point (Fig. S4). We note that this functional constraint was also present in the predecessors of this model, which were constructed to examine NO stress networks in E. coli $[36,43,66]$. Furthermore, the $P$. aeruginosa model was not trained on experimental measurements from low- $\left[\mathrm{O}_{2}\right]$ conditions, which manifest as culture densities increase. Such $\mathrm{O}_{2}$-limited conditions are of interest due to the diversity of $\mathrm{O}_{2}$ levels encountered by $P$. aeruginosa during infections, and augmenting the model to account for those environmental conditions represents an area of future growth for this research.

For $\sim 1 \mathrm{~h}$ after DPTA treatment, $\left[\mathrm{NO}_{2}{ }^{-}\right]$remained negligible, whereas $\left[\mathrm{NO}_{3}{ }^{-}\right]$increased rapidly to $43.5 \pm 0.6 \mu \mathrm{M}$ (Fig. 4A). This behavior was consistent with NO consumption being dominated by $\mathrm{NO}$ dioxygenase (Fhp), the main product of which is $\mathrm{NO}_{3}{ }^{-}$. The negligible production of $\mathrm{NO}_{2}{ }^{-}$during this period was attributed to the rapid consumption of $\mathrm{NO}$ by Fhp, which prevented appreciable $\mathrm{NO}_{2}{ }^{-}$generation from autoxidation, and the absence of NAR activity, which reduces $\mathrm{NO}_{3}{ }^{-}$to $\mathrm{NO}_{2}{ }^{-}$. Inspection of the corresponding $\mathrm{WT}\left[\mathrm{O}_{2}\right]$ dynamics revealed a brief $\sim 0.25 \mathrm{~h}$ period of NO-induced respiratory arrest, followed by resumption of $\mathrm{O}_{2}$ consumption upon clearance of NO from the culture media. Despite having the culture open to the ambient air, the respiratory activity was sufficient to deplete $\mathrm{O}_{2}$ by $1 \mathrm{~h}$ post-DPTA treatment, stabilizing at $\sim 3 \mu \mathrm{M} \mathrm{O}_{2}$. The depletion of $\mathrm{O}_{2}$ from the culture coincided with a decrease in 
$\left[\mathrm{NO}_{3}{ }^{-}\right]$, and an increase in $\left[\mathrm{NO}_{2}{ }^{-}\right]$, indicating the initiation of NAR activity. Within an hour after NAR activity was observable, the concentration of $\mathrm{NO}_{2}{ }^{-}$rapidly decreased, which suggested that NirBD and/or NirS was active. However, this rapid consumption of $\mathrm{NO}_{2}{ }^{-}$was not matched by concurrent increases in $\mathrm{NO}$ or $\mathrm{NO}_{3}{ }^{-}$, which suggested that either $\mathrm{NO}_{2}{ }^{-}$was being converted by NirBD to $\mathrm{NH}_{3}$ and/or the $\mathrm{NO}$ generated by NirS was being detoxified by NorCB, which generates $\mathrm{N}_{2} \mathrm{O}$.

\subsection{Genetic investigation of $\mathrm{NO}, \mathrm{NO}_{3}{ }^{-}$, and $\mathrm{NO}_{2}{ }^{-}$dynamics}

We sought to determine the enzymes responsible for $\mathrm{NO}, \mathrm{NO}_{3}{ }^{-}$, and $\mathrm{NO}_{2}{ }^{-}$reduction in NO-stressed $P$. aeruginosa cultures, which concomitantly would identify whether NirBD or NorCB were preventing the NO cycle. Given that the only appreciable source of $\mathrm{NO}_{3}{ }^{-}$could be Fhp, we hypothesized that negligible $\mathrm{NO}_{3}{ }^{-}$production would be observed in a $\Delta$ fhp culture treated with DPTA. As expected, when $\Delta f h p$ cultures were treated with DPTA, only trace levels of $\mathrm{NO}_{3}{ }^{-}$were observed, which confirmed that Fhp was the primary source of $\mathrm{NO}_{3}{ }^{-}$under these conditions (Fig. 4B). Notably, $\mathrm{NO}_{2}{ }^{-}$was generated at a rate similar to that observed in the absence of cells (Fig. S5), which suggested an active autoxidation pathway. Unlike WT, $\Delta f h p$ did not exhibit rapid $\mathrm{NO}_{2}^{-}$reductase activity, where only a modest decrease in $\left[\mathrm{NO}_{2}^{-}\right]$relative to the cell-free control was observed at later times (final $\left[\mathrm{NO}_{2}{ }^{-}\right]=51.7 \pm 0.6 \mu \mathrm{M}$ for $\Delta f h p$ compared to the $58.9 \pm 1.3 \mu \mathrm{M}$ measured in the absence of cells). This could have resulted from NO-mediated inhibition of NIR [67], given that NO was cleared much slower from the $\Delta f h p$ culture than WT (> $2 \mathrm{~h}$ vs. $\sim 0.25 \mathrm{~h}$, respectively), or the higher $\left[\mathrm{O}_{2}\right]$ in the $\Delta f h p$ culture, which could inhibit NIR expression and/or activity [61]. Further, since NIR expression is partially activated by $\mathrm{NO}_{3}{ }^{-}$ 
[59,61], the lack of $\mathrm{NO}_{3}{ }^{-}$may have contributed to decreased NIR activity. Collectively, the results from NO-stressed $\Delta f h p$ demonstrated that Fhp was the source of $\mathrm{NO}_{3}{ }^{-}$.

In WT cultures, consumption of $\mathrm{NO}_{3}{ }^{-}$coincided with depletion of $\mathrm{O}_{2}$ (Fig. 4A). Given that NAR activity in $P$. aeruginosa is known to function primarily under low- $\mathrm{O}_{2}$ conditions $[58,59,61]$, we reasoned that one or more of the P. aeruginosa NARs were responsible for the observed $\mathrm{NO}_{3}{ }^{-}$consumption. To determine the enzyme(s) contributing to the measured $\mathrm{NO}_{3}{ }^{-}$ consumption activity, DPTA treatment assays were conducted with $\Delta n a r G$, which inactivates a membrane-bound cytoplasmic $\mathrm{NO}_{3}{ }^{-}$reductase, and $\triangle n a p A$, which inactivates a periplasmic $\mathrm{NO}_{3}{ }^{-}$ reductase $[59,61,64,68,69]$. Deletion of napA had a negligible effect on all measured metabolites (Fig. S6), whereas loss of $\mathrm{NarG}$ eliminated virtually all $\mathrm{NO}_{3}{ }^{-}$consumption (Fig. 4C). Consistent with the loss of $\mathrm{NO}_{3}{ }^{-}$consumption in $\triangle n a r G$ cultures, the corresponding production of $\mathrm{NO}_{2}{ }^{-}$was eliminated. This confirmed that the sharp increase in $\left[\mathrm{NO}_{2}^{-}\right]$observed in DPTA-treated WT cultures at $\sim 1$ h post dose was caused by NAR, and attributed the effect specifically to NarGHI ( $\left[\mathrm{NO}_{2}{ }^{-}\right]$dynamics of $\triangle$ napA were indistinguishable from those of WT).

With Fhp and NarGHI confirmed to be the respective $\mathrm{NO}_{3}{ }^{-}$and $\mathrm{NO}_{2}{ }^{-}$sources in NOstressed $P$. aeruginosa, we sought to identify whether NirBD or NirS was responsible for $\mathrm{NO}_{2}{ }^{-}$ consumption. If $\mathrm{NirS}$ were found to contribute to $\mathrm{NO}_{2}{ }^{-}$consumption, it would mean that $\mathrm{NO}$ was only temporarily deactivated to arise again later, whereas if NirBD were the major consumption pathway, NO detoxification by Fhp would be the first step in a non-cyclic NO deactivation pathway. The rapid decrease in $\left[\mathrm{NO}_{2}{ }^{-}\right]$during the WT DPTA treatment assay confirmed the presence of NIR activity, but held no information regarding the relative contributions of NirBD and NirS. To assess what portion of the observed NIR activity could be attributed to NirBD and NirS, DPTA treatment assays were conducted with $\triangle$ nirS and $\triangle$ nirB mutants. Deletion of nirB 
had a negligible effect on the measured $[\mathrm{NO}],\left[\mathrm{O}_{2}\right],\left[\mathrm{NO}_{2}{ }^{-}\right]$, and $\left[\mathrm{NO}_{3}{ }^{-}\right]$compared to WT (Fig. S6), suggesting that NirBD was either not expressed or was inactive under the conditions studied. Conversely, $\mathrm{NO}_{2}{ }^{-}$consumption in the $\Delta n i r S$ culture was almost entirely eliminated, where $\left[\mathrm{NO}_{2}{ }^{-}\right]$peaked similarly to WT at $\sim 2 \mathrm{~h}$ post-DPTA dose, but remained steady at $\sim 66 \mu \mathrm{M}$ for the remainder of the assay (Fig. 4D). These data suggest that $P$. aeruginosa detoxifies NO with Fhp, only to have it appear again at a later time (when NirS reduces $\mathrm{NO}_{2}^{-}$to $\mathrm{NO}$ ). Further, the $\Delta$ nirS data in conjunction with that of WT, which showed that negligible amounts of NO, $\mathrm{NO}_{3}{ }^{-}$, or $\mathrm{NO}_{2}{ }^{-}$were present after $2.5 \mathrm{~h}$ (Fig. 4A), suggested that $\mathrm{NO}$ was being siphoned from the cycle and prevented from entering a second round of deactivation by Fhp. Possible pathways that could remove NO from the cycle were exchange with the gas phase, which was not rapid enough to explain the quantitative losses of $\mathrm{NO}, \mathrm{NO}_{3}{ }^{-}$, and $\mathrm{NO}_{2}{ }^{-}$(given a $k_{\mathrm{L}} a_{\mathrm{NO}}$ of $8.6 \mathrm{~h}^{-1}$ and $[\mathrm{NO}]<1 \mu \mathrm{M}$ in the culture, the amount of $\mathrm{NO}_{2}{ }^{-}$that could be lost as NO to the gas phase over a span of 30 min would be under $1 \mu \mathrm{M} \times 8.6 \mathrm{~h}^{-1} \times 0.5 \mathrm{~h}=4.3 \mu \mathrm{M} \mathrm{NO}$, which is an order of magnitude less than the observed $\sim 50 \mu \mathrm{M} \mathrm{NO}_{2}{ }^{-}$decrease), or NorCB, which reduces $\mathrm{NO}$ to $\mathrm{N}_{2} \mathrm{O}$.

In general, NORs require anaerobic or low- $\mathrm{O}_{2}$ environments to function, as $\mathrm{O}_{2}$ impairs their activity $[26,64,70]$. This is consistent with the initial DPTA-treatment assays conducted to validate the model predictions, where $\Delta n o r C$ NO consumption was indistinguishable from that of WT during the first $0.5 \mathrm{~h}$ post-DPTA dose (Fig. 2). However, after $1 \mathrm{~h}$ the $\left[\mathrm{O}_{2}\right]$ was $\sim 3 \mu \mathrm{M}$, which are conditions more conducive to NOR activity. To assess whether NorCB prevented NO cycling, DPTA treatment assays were performed with $\triangle$ norC. During the first hour following DPTA treatment, $\triangle$ norC behaved identically to WT (Fig. 4E), which was expected given that the dissolved $\left[\mathrm{O}_{2}\right]$ had just reached a low level and $\mathrm{NO}_{2}{ }^{-}$had yet to be appreciably consumed. However, approximately 15 minutes later, the [NO] dynamics of $\Delta n o r C$ cultures began to 
deviate from that of WT, exhibiting sustained oscillations of increasing amplitude (Fig. 4E). The [NO] oscillations continued for the remaining duration of the assay (3.5 $\mathrm{h}$ post-DPTA treatment), at which point the amplitude had reached the initial peak height of $\sim 3 \mu \mathrm{M}$ NO. Similar oscillations were observed in $\left[\mathrm{O}_{2}\right]$, shifted slightly out of phase with those of [NO] (Fig. S7A), which was consistent with a previously-identified oscillatory mechanism driven by a competition between NO dioxygenase and respiratory cytochromes over the limited dissolved $\mathrm{O}_{2}$ in the system [43]. Further, $\left[\mathrm{NO}_{2}^{-}\right]$in the $\Delta$ norC culture did not drop as rapidly as WT, and the $\left[\mathrm{NO}_{3}{ }^{-}\right]$ began increasing in $\Delta$ norC at the time when $\left[\mathrm{NO}_{2}{ }^{-}\right]$declined, whereas $\left[\mathrm{NO}_{3}{ }^{-}\right]$remained at undetectable levels in WT cultures after its initial clearance. These data confirmed that NorCB was the major pathway siphoning NO from the NO cycle prior to its second deactivation by Fhp.

\subsection{Analysis of $\mathrm{NO}$ cycle initiated by $\mathrm{NO}_{3}{ }^{-}$}

Results presented in Figure 4 suggest that $P$. aeruginosa initially detoxifies $\mathrm{NO}$ to $\mathrm{NO}_{3}{ }^{-}$ only to have it arise again later from $\mathrm{NO}_{2}{ }^{-}$. Within the assays presented thus far, $\mathrm{NO}$ is delivered to cultures by release from DPTA. In consideration of the kinetic release rate of DPTA, only 30 $\mu \mathrm{M}$ of NO would have been left to be released from DPTA by 1.5 hours post-dose, which corresponded to the time that NO briefly returned to WT cultures and $\Delta n o r C$ began exhibiting NO oscillations. Given this calculation, data from $\Delta n i r S$, and the magnitude of NO oscillations in

$\Delta$ nor $C$, it is reasonable to assume that $\mathrm{NO}_{2}^{-}$consumption sustained the $\mathrm{NO}$ cycle and oscillations in $\Delta$ norC. However, to provide further experimental evidence that the NO cycle is driven by endogenous NO production, cultures of WT and $\Delta n o r C P$. aeruginosa were treated with a single bolus of $100 \mu \mathrm{M} \mathrm{NO}_{3}{ }^{-}$(final concentration) at time zero (DPTA was not present), and the resulting $[\mathrm{NO}],\left[\mathrm{O}_{2}\right],\left[\mathrm{NO}_{3}{ }^{-}\right]$, and $\left[\mathrm{NO}_{2}^{-}\right]$were monitored for $3.5 \mathrm{~h}$ (Fig. 5). 

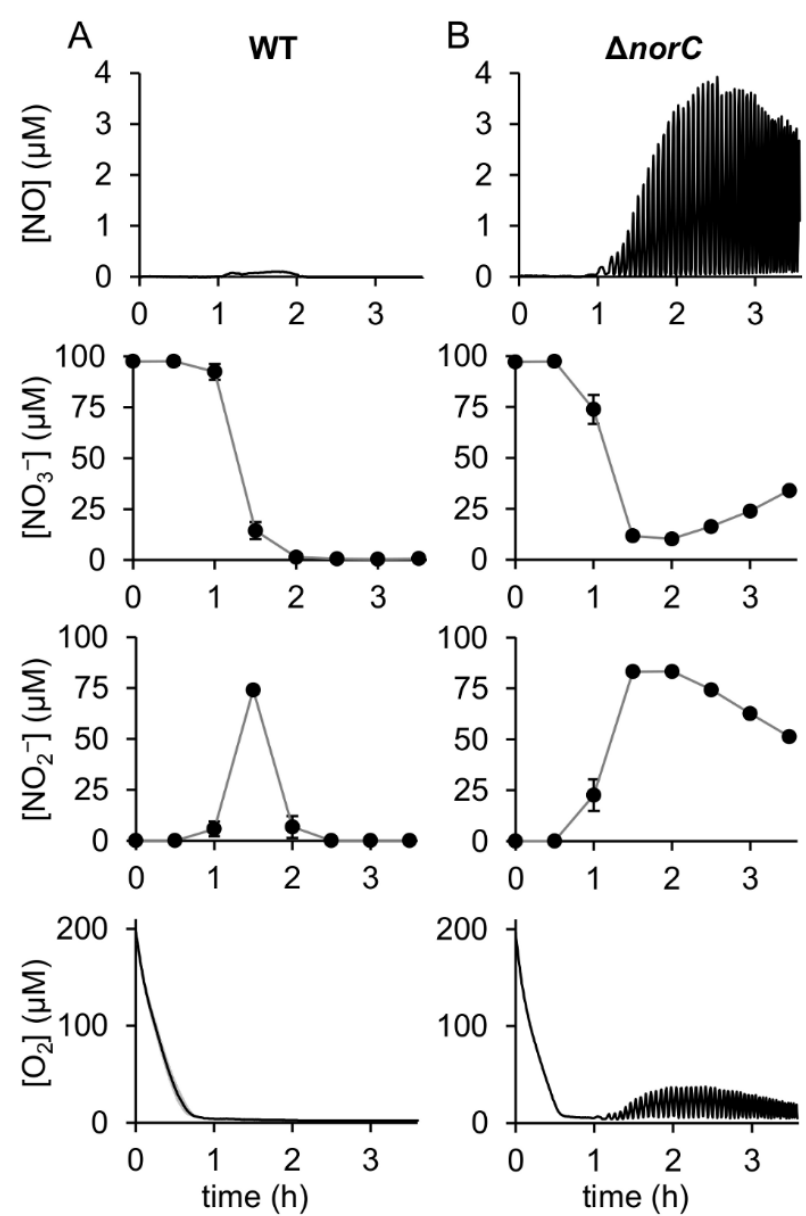

Figure 5. Metabolic cycling of $\mathrm{NO}$ following treatment with $\mathrm{NO}_{3}{ }^{-}$. (A) WT and (B) $\triangle$ norC PAO1 cultures at an initial $\mathrm{OD}_{600}$ of 0.05 were treated with $100 \mu \mathrm{M} \mathrm{NaNO}_{3}$ at time zero, and monitored for $3.5 \mathrm{~h}$ post-dose.

Concentrations of $\mathrm{NO}$ and $\mathrm{O}_{2}$ were measured continuously, whereas $\mathrm{NO}_{2}{ }^{-}$and $\mathrm{NO}_{3}{ }^{-}$were quantified every $0.5 \mathrm{~h}$. Solid black lines (or circles) are the mean of 3 independent experiments, with gray shading (or error bars) representing the SEM. The exception is $[\mathrm{NO}]$ and $\left[\mathrm{O}_{2}\right]$ of $\triangle n o r C$, which are data from one representative experiment because averaging multiple experiments obscures the oscillatory dynamics (see Fig. S10 for remaining $\Delta$ norC data).

For both strains, within the first $0.5 \mathrm{~h}$ of $\mathrm{NO}_{3}{ }^{-}$addition, there was no change in any of the measured metabolite concentrations except $\mathrm{O}_{2}$, which was steadily depleted from the cultures. By $1 \mathrm{~h}$ post-dose, the $\left[\mathrm{O}_{2}\right]$ had stabilized at $\sim 3 \mu \mathrm{M}\left(\sim 1.5 \%\right.$ of the air-saturated $\mathrm{O}_{2}$ concentration), and NAR activity began converting the $\mathrm{NO}_{3}{ }^{-}$to $\mathrm{NO}_{2}{ }^{-}$. Flux through the $\mathrm{NO}_{3}{ }^{-}$to $\mathrm{NO}_{2}{ }^{-}$arm of the cycle increased considerably between 1 and $1.5 \mathrm{~h}$, as illustrated by large drops in $\left[\mathrm{NO}_{3}{ }^{-}\right]$and concurrent increases in $\left[\mathrm{NO}_{2}{ }^{-}\right]$for both strains. The rate of $\mathrm{NO}_{2}{ }^{-}$consumption greatly increased over the next $0.5 \mathrm{~h}$ for WT, where $\left[\mathrm{NO}_{2}{ }^{-}\right]$decreased to $\sim 7 \mu \mathrm{M}$ at $2 \mathrm{~h}$ post- $\mathrm{NO}_{3}{ }^{-}$ 
treatment. Consistent with results from DPTA treatments, consumption of $\mathrm{NO}_{2}{ }^{-}$did not coincide with a comparable increase in $\mathrm{NO}$ for $\mathrm{WT}$, and neither $\mathrm{NO}, \mathrm{NO}_{3}{ }^{-}$, nor $\mathrm{NO}_{2}{ }^{-}$were detected at later time points. However, unlike WT, with $\Delta$ norC $\mathrm{NO}$ became detectable when $\mathrm{NO}_{2}{ }^{-}$began to accumulate, and its concentration oscillated (Fig. 5B). Oscillations in $\left[\mathrm{O}_{2}\right]$ that were slightly offphase with [NO] were observed (Fig. S7B), $\mathrm{NO}_{2}{ }^{-}$consumption was slower than that of WT, and $\mathrm{NO}_{3}{ }^{-}$began to accumulate in $\triangle$ nor $C$ cultures. These data provide additional evidence that NO cycles in $P$. aeruginosa cultures when NorCB activity is absent, and that it is endogenously generated NO that contributes to this process.

\subsection{Analysis of the period and amplitude of NO oscillations}

The metabolic oscillations reported here for $\Delta$ nor $C$ cultures of $P$. aeruginosa resemble those of a previous study we performed on microaerobic NO detoxification [43]. However, there are notable differences in the oscillation dynamics, especially with regard to amplitude and period. For example, from our previous study, the period and amplitude of the largest NO oscillation were approximately $10 \mathrm{~min}$ and $0.5 \mu \mathrm{M}$ in E. coli populations cultured in environments where $10 \mu \mathrm{M}\left[\mathrm{O}_{2}\right]$ was the concentration in equilibrium with the surrounding atmosphere, whereas the period and amplitude of the largest oscillation in $\Delta$ norC P. aeruginosa populations cultured here (equilibrium $\left[\mathrm{O}_{2}\right]$ of $210 \mu \mathrm{M}$ ) was approximately 3 min and $3.4 \mu \mathrm{M}$, respectively. Further, in our previous study, we observed NO oscillations in WT P. aeruginosa populations cultured in environments where $50 \mu \mathrm{M}\left[\mathrm{O}_{2}\right]$ was the concentration in equilibrium with the surrounding atmosphere, and the period and amplitude of the largest oscillation was approximately 4 minutes and $0.35 \mu \mathrm{M}$. Since these differences in oscillation dynamics could be attributed to environmental or bacteria-associated factors, we sought to identify those variables 
with considerable influence on the oscillation properties. To do this, we turned to a minimal NO model that displays these metabolic oscillations from our previous study, and analyzed how varying the magnitude of parameters associated with environmental and bacteria-specific differences alter the period and amplitude of oscillations. Both E. coli and P. aeruginosa possess the necessary genetic components for oscillatory behavior $\left(\mathrm{NOD}\right.$ and $\mathrm{O}_{2}$-consuming respiratory cytochromes [43]), but they differ in the number of these components (E. coli harbors three cytochromes, whereas $P$. aeruginosa has five $[53,61,62])$, as well as in the amino acid sequence and catalytic activity of their respective NODs [71]. The minimal model used for the oscillatory analysis is presented in Model S2.

To begin, we investigated how environmental variables affected oscillation dynamics, and focused specifically on the rate of NONOate disassociation and dissolved $\left[\mathrm{O}_{2}\right]$ that was in equilibrium with the surrounding atmosphere. The NONOate disassociation rate dictated the delivery of $\mathrm{NO}$ to cultures, and it is a function of $\mathrm{pH}$, which was different between media used for $E$. coli and $P$. aeruginosa $(\mathrm{pH}=7.4$ for $E$. coli MOPS media, $\mathrm{pH}=7.0$ for $P$. aeruginosa/BSM media). Interestingly, when this parameter was varied over a 4-fold range from its original value, a non-monotonic response was observed, with period and amplitude initially increasing as $\mathrm{NO}$ was delivered faster, only to decrease at a higher release rate (Fig. 6A). The $\left[\mathrm{O}_{2}\right]$ in equilibrium with the surrounding atmosphere affects $\mathrm{O}_{2}$ transport into the culture and the maximum $\left[\mathrm{O}_{2}\right]$ that can be attained, and previous observations have shown that this variable has the capacity to eliminate or enable oscillations [43]. When the equilibrium $\left[\mathrm{O}_{2}\right]$ was varied within a 4-fold range, higher values produced oscillations with a shorter period and amplitude than those at lower values (Fig. 6B). Taken together, these simulation results suggest that the 
NONOate disassociation rate and equilibrium $\left[\mathrm{O}_{2}\right]$ exert considerable influence on the $\mathrm{NO}$

oscillation period and amplitude.

A environmental parameters

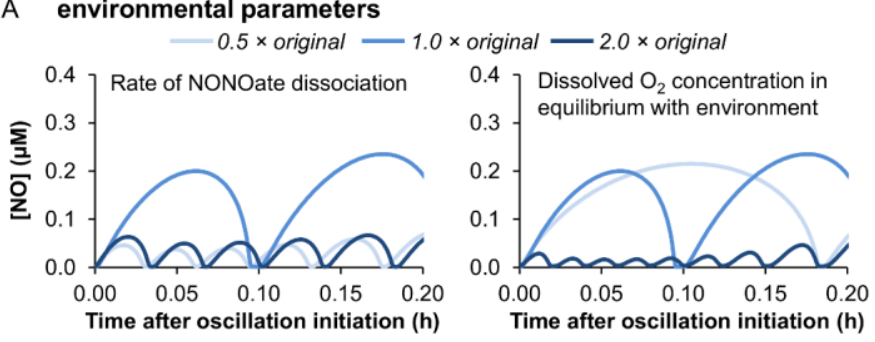

B cellular parameters (enzyme affinities)
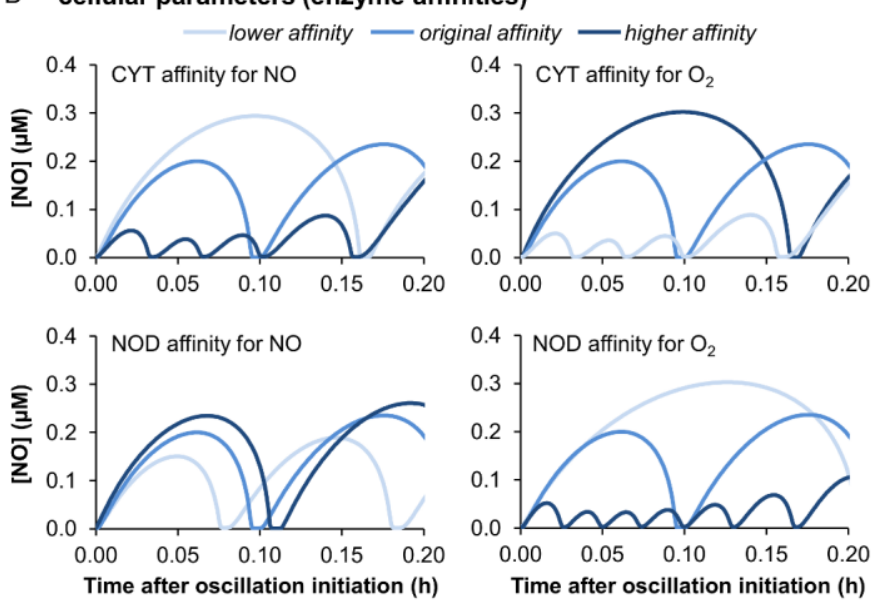

C cellular parameters (NOD inhibition and NOR addition)

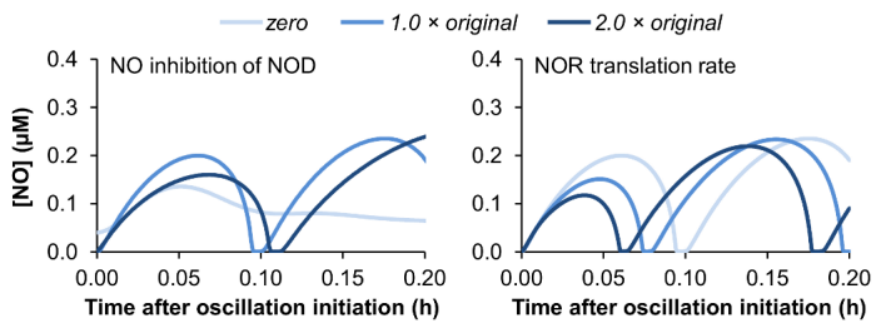

Figure 6. Impact of environmental and cellular parameters on NO oscillation dynamics. The core oscillatory model from [43] was used to simulate NO treatment and assess the effect of varying key model parameters on the resulting oscillations. To facilitate comparison of oscillations, the time-scale ( $x$-axis) was shifted to reflect the time after oscillations began, rather than time after NONOate treatment. (A) Parameters related to culturing conditions were increased and decreased by two-fold of their original value: NONOate release rate $\left(k_{\text {NONOate }}\right)$ and dissolved $\left[\mathrm{O}_{2}\right]$ in equilibrium with the environment $\left(\left[\mathrm{O}_{2}\right]_{\text {env }}\right)$. (B) Cellular parameters governing the binding affinity of NOD and CYT (high-affinity cytochrome) for $\mathrm{NO}$ and $\mathrm{O}_{2}$ were varied within two-fold of their original value: on-rate of $\mathrm{NO}$ to CYT $\left(k_{\mathrm{on}, \mathrm{NO}}\right), \mathrm{O}_{2}$ Michaelis constant for CYT $\left(K_{\mathrm{m}, \mathrm{O} 2}\right)$, on-rate of NO to $\mathrm{O}_{2}$-bound NOD $\left(k_{\mathrm{ox}}\right)$, and on-rate of $\mathrm{O}_{2}$ to NOD $\left(k_{\mathrm{O} 2}\right)$. (C) Cellular parameters governing NO-mediated NOD inhibition $\left(k_{\mathrm{On}, \mathrm{NO}}\right)$ and the expression of NOR $\left(k_{\text {translate, NOR }}\right)$ were set to zero, their original value, or increased two-fold from their original value. Since NOR was not originally present in the core oscillatory model, the "original" value is derived from the full E. coli model. The core model, which includes the "original" values of all parameters described here, are presented in Model S2. 
To explore variables that can vary between species or strains, we investigated parameters governing the activity of the respiratory cytochromes and NOD (Fig. 6B). For the cytochromes, we observed that increasing the affinity for $\mathrm{O}_{2}\left(e . g\right.$., decreasing associated $\left.K_{\mathrm{m}}\right)$ increased the oscillation period and amplitude, whereas increasing the affinity for NO had the opposite effect. This can be rationalized by considering that $\mathrm{O}_{2}$ and $\mathrm{NO}$ compete with one another for binding to the cytochromes, and the cytochromes outcompete NOD for $\mathrm{O}_{2}$. With respect to NOD, increasing its affinity for NO increased the amplitude and period, whereas increasing its affinity for $\mathrm{O}_{2}$ had the opposite effect. For $\mathrm{O}_{2}$, increasing its affinity enables NOD to compete more effectively with the cytochromes for this common substrate, which one would expect to decrease the oscillation amplitude and period. The NOD affinity for NO in this context refers to the binding of $\mathrm{NO}$ with the $\mathrm{O}_{2}$-bound form of $\mathrm{NOD}$, leading to $\mathrm{NO}_{3}{ }^{-}$product formation, rather than the binding of NO to NOD prior to $\mathrm{O}_{2}$, which is a process known in E. coli to inhibit NOD activity [72]. The increase in oscillation period and amplitude observed upon increasing NO affinity of NOD can be explained by the faster rate at which $\mathrm{O}_{2}$-bound NOD is consumed, leaving more unbound NOD exposed for potential inhibition by NO. This parameter is therefore indirectly increasing the rate of NO-mediated NOD inhibition, resulting in larger [NO] peaks from which the bacteria are slower to recover.

Given that NO-mediated inhibition of NOD appeared to have an effect on oscillation dynamics, and because this inhibitory reaction has not yet been reported for the NOD of $P$. aeruginosa, Fhp, we explored a parameter directly governing this process $\left(k_{\mathrm{on}, \mathrm{NO}}\right.$, the rate of inhibitory binding of NO to NOD). The NO binding rate was increased by two-fold and decreased to zero, to represent the possible absence of this process in P. aeruginosa (Fig. 6C). Interestingly, the oscillations were eliminated when the rate of NO binding was set to zero, 
suggesting that this inhibitory effect may play an important role in the oscillation mechanism.

Since $P$. aeruginosa cultures were shown experimentally to exhibit oscillations, we hypothesize that, similar to E. coli Hmp, P. aeruginosa Fhp also experiences NO-mediated substrate inhibition; however, this effect requires experimental verification with biochemical assays. Another important distinction between the previously observed oscillations and those reported here was the required deletion of NOR under the conditions investigated in this study. We therefore examined the effect of varying flux through a NOR reaction on the oscillation dynamics. Since NOR was absent from the minimal oscillation model, it was introduced by transferring all NOR-related reactions, parameters, and species from the full E. coli model [43] to the minimal oscillating model. Flux through NOR was varied by changing the rate of NOR expression $\left(k_{\text {translate,NOR }}\right)$ from zero to the original parameter value, and two-fold the original value (Fig. 6C). Increasing rates of NOR expression resulted in decreased oscillation amplitude and period, which is expected given that this enzyme serves as an additional NO sink. To provide additional experimental confirmation that NOR repress NO oscillations, we performed experiments with $E$. coli lacking NOR ( $\Delta$ norV) under the same conditions as used in our previous study (50 $\mu \mathrm{M}$ DPTA NONOate, $10 \mu \mathrm{M} \mathrm{O}_{2}$ environment) [43]. As predicted, the resulting oscillations within $\Delta n o r V E$. coli cultures exhibited an increased period and amplitude relative to WT (approximately $14 \mathrm{~min}$ and $0.7 \mu \mathrm{M}$, respectively, for the maximum oscillation, a $40 \%$ increase over the $10 \mathrm{~min}$ and $0.5 \mu \mathrm{M}$ of WT) (Fig. S8). The considerable impact many of the parameters analyzed here had on the period and amplitude of oscillations suggests that these features should be expected to vary as a function of bacterial species and culturing conditions. 


\section{DISCUSSION}

P. aeruginosa are prevalent, opportunistic pathogens that are increasingly refractory to many antibiotics $[5,6,73]$. Cystic fibrosis patients commonly develop P. aeruginosa infections within their airways, nosocomial infections (e.g., ventilator-associated pneumonia) are often caused by $P$. aeruginosa, and $P$. aeruginosa is a frequent inhabitant of chronic, non-healing wounds $[1,2,74]$. The propensity for $P$. aeruginosa to form biofilms combined with both its innate and acquired resistance toward antibiotics prompted the Center for Disease Control to label multi-drug resistant $P$. aeruginosa as a "serious threat" to public health [75]. To address this threat, novel treatment modalities are needed [6,76], and one of those being explored focuses on $\mathrm{NO}[28,77-80]$.

Due to the ability of NO to disperse $P$. aeruginosa biofilms and the importance of NO detoxification machinery to $P$. aeruginosa virulence [20,81], several groups have investigated the use of $\mathrm{NO}$ as an alternative to conventional antibiotics [77-80]. Miller and colleagues observed a significant reduction in $P$. aeruginosa bacterial load in a rat model of cystic fibrosis with the use of inhaled, gaseous NO when compared to untreated controls [79]. In a phase I clinical trial, gaseous NO reduced bacterial loads, which included $P$. aeruginosa, within the sputum of cystic fibrosis patients [77]. In addition, Friedman and colleagues observed impressive growth inhibition of numerous Gram-positive and Gram-negative clinical isolates, which included $P$. aeruginosa, when NO-delivering nanoparticles were used in comparison to control particles [78].

Bacterial NO stress networks are dominated by enzymes that rapidly consume NO, and thereby restrict its reactions with other pathways, such as those that lead to protein and DNA damage or metabolic dysfunction. In essence, this is analogous to substrates (e.g., NO) failing to 
be converted into a desirable product (e.g., inactive, nitrosylated Fe-S clusters) due to competing reactions (e.g., detoxification by NO dioxygenase). To modulate such native pathways for the improvement of cellular properties, as is a typical endeavor in metabolic engineering, understanding how systems quantitatively function is enabling. In the case of $\mathrm{NO}$ and $P$. aeruginosa, previous studies have clearly linked this metabolite and its biochemical network to the pathogen's virulence $[20,28,29]$, and in the context of health and disease, a desirable cellular property would be limited NO flux through detoxification pathways, and increased flux through pathways that damage the pathogen. To enable such interventions, quantitative knowledge of $P$. aeruginosa NO metabolism is desirable much in the same way that quantitative knowledge of substrate metabolism is desirable for rational improvements to the production of chemicals.

Given the critical role of NO in the pathogenicity and biofilm life-style of $P$. aeruginosa, as well as its potential to treat $P$. aeruginosa infections [77-80], we constructed a kinetic model of the $P$. aeruginosa NO biochemical network. The model is a computational tool that enables NO distributions in P. aeruginosa cultures to be quantitatively analyzed (Fig. 3), and previous models of this type have been constructed for $E$. coli $\mathrm{K}-12$ and enterohemorrhagic $E$. coli O157:H7 [36,60]. The utility of these models has been demonstrated by uncovering a novel kinetic regime in which NO detoxification machinery performs poorly [36], predicting dosing regimens that maximize bacteriostasis at prescribed NO payloads [42], identifying the mechanism through which loss of $\mathrm{ClpP}$ impairs E. coli NO defenses [66], and delineating the subnetwork responsible for $\mathrm{NO}$ and $\mathrm{O}_{2}$ oscillations within microaerobic environments [43]. We anticipate that similar advances will be enabled by the $P$. aeruginosa NO model described here, and importantly, translation of the modeling approach we employed, which thus far has only 
been demonstrated in E. coli strains [60], to a non-model organism is an important milestone for broader application of the methodology.

Using the model developed in this study, we sought to determine whether NO was cycled in $P$. aeruginosa cultures. We were intrigued by the possibility that NO would be temporarily deactivated only to arise again at a later time, and there is evidence that $\mathrm{NO}, \mathrm{NO}_{3}^{-}$, and $\mathrm{NO}_{2}{ }^{-}$, which comprise the metabolites of this potential cycle, are present in the lungs of cystic fibrosis patients [32,50]. Further, this NO cycle appears to be broadly distributed, where 217 species within the KEGG database possess the enzymatic capacity to execute such a cycle (NO dioxygenase, $\mathrm{NO}_{3}{ }^{-}$reductase, and NO-generating $\mathrm{NO}_{2}{ }^{-}$reductase) (Table S7) [62]. Recently, data supporting the existence of NO cycles in Corneybacterium glutamicum [82] and Streptomyces coelicolor [83] have been obtained. In addition, NO cycling has been known to occur in plants [84] and mammals [85], of which the latter includes the function of NO synthases. Computational analysis of NO detoxification by aerobic $P$. aeruginosa cultures (Fig. 3), which were used to ensure Fhp activity, indicated a metabolic block in the cycle at $\mathrm{NO}_{3}{ }^{-}$. That block was relieved as $P$. aeruginosa consumed $\mathrm{O}_{2}$ to drive metabolism into a microaerobic regime, within which $\mathrm{NO}_{3}{ }^{-}$was converted into $\mathrm{NO}_{2}{ }^{-}$, and $\mathrm{NO}_{2}{ }^{-}$was consumed without production of any appreciable $\mathrm{NO}$ or $\mathrm{NO}_{3}{ }^{-}$(Fig. 4A). Using a genetic approach, we identified NorCB as the enzyme drawing flux out of the NO cycle, and in its deletion strain, $\Delta n o r C$, high frequency oscillations in $[\mathrm{NO}]$ and $\left[\mathrm{O}_{2}\right]$ were observed. These oscillations, which resemble those that we previously observed for NO-stressed P. aeruginosa in low $\mathrm{O}_{2}$ environments [43], are indicative of an unbalanced competition for $\mathrm{O}_{2}$ between Fhp and the aerobic terminal oxidases.

$\mathrm{NO}$ is a metabolite with many functions, which range from neuronal signaling and vasodilation to use as an antimicrobial by immune cells [86-88]. NO also has an important role 
in the nitrogen cycle as an intermediate in denitrification $[64,89]$. At the intersection of pathogenesis and denitrification lies $P$. aeruginosa, which is a dangerous human pathogen that can perform denitrification. $P$. aeruginosa can generate NO, is attacked by $\mathrm{NO}$, and uses $\mathrm{NO}$ as a signal for virulence factor expression and biofilm dispersal [29,31,90]. Collectively, the work presented here provides a computational tool for the quantitative analysis of NO metabolism in $P$. aeruginosa, and experimental evidence establishing that $P$. aeruginosa prevents NO cycling with the use of NO reductase. In this study, planktonic cultures of P. aeruginosa were used, and

there is certainly precedent for knowledge gained from planktonic investigations to be important within the biofilm lifestyle (for example, see [91,92]). However, biofilms are far more complex, for example with the presence of nutrient gradients and significant growth heterogeneity, as compared to their planktonic counterparts, and it will be exciting to see how $P$. aeruginosa NO metabolism within these lifestyles quantitatively compares as the approaches used here are adapted and applied to biofilms in future research. We postulate that as more quantitative knowledge of $P$. aeruginosa NO metabolism is gained, such understanding in time, could be translated into the development of novel anti-infectives for treatment of infections caused by this prevalent pathogen.

\section{MATERIALS AND METHODS}

\subsection{Bacterial strains and knockout construction}

\subsubsection{Bacterial strains}

P. aeruginosa PAO1 (ATCC 15692) was used in this study, and is referred to as the WT strain. Single gene deletion mutants were constructed from the WT PAO1 parent strain to inactivate $\mathrm{NO}$ dioxygenase $(\Delta f h p), \mathrm{NO}$ reductase $(\Delta n o r C)$, assimilatory $\mathrm{NO}_{2}{ }^{-}$reductase $(\Delta n i r B)$, 
dissimilatory $\mathrm{NO}_{2}{ }^{-}$reductase $(\Delta n i r S)$, periplasmic $\mathrm{NO}_{3}{ }^{-}$reductase $(\Delta n a p A)$, or membrane-bound $\mathrm{NO}_{3}{ }^{-}$reductase $(\Delta n a r G)$. The strain of $E$. coli used in this study was a $\Delta n o r V$ mutant (lacking NO reductase) of MG1655, constructed in a previous study [36].

\subsubsection{Construction of knockout mutants}

P. aeruginosa mutants were constructed using the lambda Red recombinase system [93] to replace the target gene with a gentamicin resistance cassette (harboring gentamicin 3'acetyltransferase; $a a c C 1$ ), abbreviated here as $\mathrm{gm}^{\mathrm{R}}$. Briefly, the $\mathrm{gm}^{\mathrm{R}}$ cassette was cloned from the pAS03 plasmid [94] using Phusion High-Fidelity DNA Polymerase (New England Biolabs; NEB), with primers possessing flanking 40 nt sequences that were homologous to the PAO1 genomic sequence upstream and downstream of the target gene to be deleted (see Table S8A for knockout primers used in this study). Two additional PCRs were performed to amplify $4400-600$ nt regions upstream and downstream of the target gene. An overlap extension PCR [95] was used to combine the 3 DNA fragments (the upstream and downstream homology regions, and the fragment possessing the $\mathrm{gm}^{\mathrm{R}}$ cassette), and amplify the joined product. The joined DNA product was gel-purified (Qiagen), eluted in $\mathrm{DI} \mathrm{H}_{2} \mathrm{O}$, and stored at $-20^{\circ} \mathrm{C}$.

WT PAO1 cells were made electrocompetent, and transformed (see Section 4.1.3 for further detail) with the pUCP18-RedS plasmid, which encodes for the lambda Red recombinase system [93]. Transformants were plated on LB + agar plates containing $100 \mu \mathrm{g} / \mathrm{mL}$ carbenicillin to select for positive clones. The PAO1 pUCP18-RedS cells were again made electrocompetent, except growth was conducted in the presence of $100 \mu \mathrm{g} / \mathrm{mL}$ carbenicillin, and the cells were induced with $10 \mathrm{mM} \mathrm{L-arabinose} \mathrm{overnight} \mathrm{to} \mathrm{activate} \mathrm{expression} \mathrm{of} \mathrm{the} \mathrm{lambda} \mathrm{Red} \mathrm{system.}$ Electrocompetent cells were transformed with $\geq 500 \mathrm{ng}$ of the purified DNA product, and plated 
on LB + agar plates containing $30 \mu \mathrm{g} / \mathrm{mL}$ gentamicin. Colonies were selected from the plate, and re-streaked on LB $+300 \mathrm{mM}$ sucrose plates to remove the pUCP18-RedS plasmid, where counter-selection was enabled by the presence of the $s a c B$ gene on the plasmid [93]. Positive clones were verified with colony PCR, using two sets of primers: one set with both forward and reverse primers homologous to regions within the targeted gene to confirm absence of the deleted gene in the mutant, and another with a forward primer upstream of the gene and reverse primer within the $\mathrm{gm}^{\mathrm{R}}$ cassette to confirm proper genomic location of the inserted $\mathrm{gm}^{\mathrm{R}}$ cassette (see Table S8B for the list of verification primers used in the present study).

\subsubsection{Plasmid and DNA transformation}

PAO1 cells (from $-80^{\circ} \mathrm{C}$ frozen stock) were grown for $16 \mathrm{~h}$ in $3 \mathrm{~mL}$ of $\mathrm{LB}$ at $37^{\circ} \mathrm{C}$ and $250 \mathrm{rpm}$. For a gene deletion using the PAO1 pUCP18-RedS strain, the LB also contained 100 $\mu \mathrm{g} / \mathrm{mL}$ carbenicillin, and $10 \mathrm{mM}$ L-arabinose was added after $4 \mathrm{~h}$ of growth. The overnight culture was centrifuged $(16,000 \times g)$ for $1 \mathrm{~min}$, the supernatant discarded, and the pellet resuspended in $1 \mathrm{~mL}$ of room-temperature, $300 \mathrm{mM}$ sucrose (dissolved in D.I. $\mathrm{H}_{2} \mathrm{O}$ and filtersterilized) — a solution commonly used to make $P$. aeruginosa electrocompetent $[8,93]$. The centrifugation and resuspension were repeated for 3 additional iterations, after which the pellet was resuspended in $100 \mu \mathrm{L}$ of the sucrose solution. Purified plasmid or linear DNA was added to the cellular resuspension, and mixed gently with pipetting. The mixture was transferred to a 2 mm-gap electroporation cuvette, and electroporated at $2.5 \mathrm{kV}$. Immediately after electroporation, $1 \mathrm{~mL}$ of room-temperature LB was added to the cuvette, mixed gently by pipette, and transferred to a test tube. The cells were incubated at $37^{\circ} \mathrm{C}$ and $250 \mathrm{rpm}$ for $10 \mathrm{~min}$, after which they were centrifuged and washed with fresh LB to eliminate residual sucrose, which inhibits growth of 
cells possessing the pUCP18-RedS plasmid harboring the $s a c B$ gene. Cells were again transferred to a test tube, and incubated at $37^{\circ} \mathrm{C}$ and $250 \mathrm{rpm}$ for $3 \mathrm{~h}$, after which they were plated on LB + agar plates containing the appropriate selection marker.

\subsection{Growth media and chemicals}

Rich media used in this study was LB Broth (BD Difco). The minimal media used for $P$. aeruginosa was basal salts media (BSM) $\left(30.8 \mathrm{mM} \mathrm{K}_{2} \mathrm{HPO}_{4}, 19.3 \mathrm{mM} \mathrm{KH} \mathrm{PO}_{4}, 15.0 \mathrm{mM}\right.$ $\left(\mathrm{NH}_{4}\right)_{2} \mathrm{SO}_{4}, 1 \mathrm{mM} \mathrm{MgCl}$, and $\left.2 \mu \mathrm{M} \mathrm{FeSO}_{4}\right)$ [96] supplemented with $15 \mathrm{mM}$ succinate as the sole carbon source (referred to as "BSM" throughout the text). For experiments involving E. coli, the minimal media used was MOPS [3-(N-morpholino)propanesulfonic acid] media (Teknova) with $10 \mathrm{mM}$ glucose as the sole carbon source. DPTA NONOate, (Z)-1-[N-(3-aminopropyl)-N-

(3-ammoniopropyl)amino]diazen-1-ium-1,2-diolate (Cayman Chemical), was used as a chemical NO donor, which dissociates with a half-life of approximately $1 \mathrm{~h}$ at $\mathrm{pH} 7.0$ and $37^{\circ} \mathrm{C}$ to release 2 mol of NO per mol of parent compound. Antibiotics used were gentamicin $(30 \mu \mathrm{g} / \mathrm{mL})$ and carbenicillin $(100 \mu \mathrm{g} / \mathrm{mL})$. Unless noted otherwise, all chemical and media components were purchased from Sigma Aldrich or Fisher Scientific, and were autoclaved (LB media) or filtersterilized $(0.2 \mu \mathrm{m}$ filters $)$ prior to use.

\subsection{DPTA and $\mathrm{NO}_{3}{ }^{-}$treatment assays}

One $\mathrm{mL}$ of $\mathrm{LB}$ was inoculated with a scrape of frozen $-80{ }^{\circ} \mathrm{C} \mathrm{PAO} 1$ cells, and grown overnight $(16 \mathrm{~h})$ at $37^{\circ} \mathrm{C}$ and $250 \mathrm{rpm}$. After overnight growth, the culture was mixed gently with a pipette to disrupt cell clumps, and $200 \mu \mathrm{L}$ was removed to inoculate $20 \mathrm{~mL}$ of fresh BSM in a $250 \mathrm{~mL}$ baffled shake flask. The flask culture was incubated at $37^{\circ} \mathrm{C}$ and $250 \mathrm{rpm}$ until it 
reached an $\mathrm{OD}_{600}$ of 0.2 , at which point $8 \mathrm{~mL}$ were removed and transferred to $1.5 \mathrm{~mL}$ microcentrifuge tubes. The cells were centrifuged at $17,000 \times g$ for $1 \mathrm{~min}$, the supernatant was removed, and the pellets were resuspended and combined in $1 \mathrm{~mL}$ of fresh, pre-warmed $\left(37^{\circ} \mathrm{C}\right)$ BSM. The cell resuspension was used to inoculate $10 \mathrm{~mL}$ of fresh, pre-warmed BSM in a bioreactor to an $\mathrm{OD}_{600}$ of 0.05 , after removing an equivalent volume (to maintain $10 \mathrm{~mL}$ total). Immediately after inoculation, bioreactor cultures were treated with $50 \mu \mathrm{M}$ DPTA NONOate or $100 \mu \mathrm{M} \mathrm{NaNO}$.

DPTA treatment of $\Delta n o r V E$. coli was performed identically as described previously [43]. Briefly, $1 \mathrm{~mL}$ of LB media was inoculated with a scrape from a $-80{ }^{\circ} \mathrm{C}$ frozen stock, and grown at $37^{\circ} \mathrm{C}$ and $250 \mathrm{rpm}$ for $4 \mathrm{~h} .10 \mu \mathrm{L}$ of LB culture was transferred to fresh MOPS media, and grown at $37{ }^{\circ} \mathrm{C}$ and $250 \mathrm{rpm}$ in a hypoxic chamber (Coy Laboratory Products) overnight (16 h). The chamber environment was maintained at an $\mathrm{O}_{2}$ level to achieve a dissolved $\left[\mathrm{O}_{2}\right]$ in media at equilibrium with the environment of $10 \mu \mathrm{M}$, and contained approximately $0.2 \%(2,000 \mathrm{ppm})$ $\mathrm{CO}_{2}$, and the balance $\mathrm{N}_{2}$. A $250 \mathrm{~mL}$ baffled shake-flask with $20 \mathrm{~mL}$ of fresh MOPS media was inoculated to an $\mathrm{OD}_{600}$ of 0.01 with the overnight culture, and grown at $37{ }^{\circ} \mathrm{C}$ and $250 \mathrm{rpm}$ in the chamber. Upon reaching and $\mathrm{OD}_{600}$ of 0.2 , the flask culture was used to inoculate $10 \mathrm{~mL}$ fresh MOPS media in the bioreactor (operated in the hypoxic chamber) to an $\mathrm{OD}_{600}$ of 0.05 , and the bioreactor culture was treated immediately with $50 \mu \mathrm{M}$ DPTA NONOate.

\subsection{Bioreactor apparatus}

The bioreactor apparatus used to facilitate continuous measurements of $[\mathrm{NO}]$ and $\left[\mathrm{O}_{2}\right]$ was adopted from a setup in a previous study [43], and consisted of a $50 \mathrm{~mL}$ polypropylene conical tube containing $10 \mathrm{~mL}$ of media, open to the ambient air, and magnetically stirred with 
an ethanol-sterilized (soaked in 70\%/30\% ethanol/D.I. $\mathrm{H}_{2} \mathrm{O}$ for $>1 \mathrm{~h}$ ) stir bar. The conical tube was suspended in a magnetically-stirred water bath, maintained at $37^{\circ} \mathrm{C}$ with a stirring hotplate. The apparatus in its entirety was operated within a biological safety cabinet (Class II, Type A2 Biological Safety Cabinet, Labconco), except for the experiments conducted with E. coli, for which the bioreactor apparatus was operated in a hypoxic chamber (described in the section above).

\subsection{Quantification of $\mathrm{NO}$ and $\mathrm{O}_{2}$ concentrations}

$[\mathrm{NO}]$ and $\left[\mathrm{O}_{2}\right]$ in bioreactor cultures were monitored continuously $(\geq 1 \mathrm{reads} / \mathrm{sec})$ throughout assays, using a 2 mm ISO-NOP sensor (World Precision Instruments) and a fiberoptic FireSting $\mathrm{O}_{2}$ robust miniprobe (PyroScience), respectively.

\subsection{Quantification of $\mathrm{NO}_{2}{ }^{-}$and $\mathrm{NO}_{3}{ }^{-}$concentrations}

$\left[\mathrm{NO}_{2}{ }^{-}\right]$and $\left[\mathrm{NO}_{3}{ }^{-}\right]$were measured using the Nitrate/Nitrite Colorimetric Assay Kit (Cayman Chemical), which is based on a Griess assay. Samples of $300 \mu \mathrm{L}$ were removed from the culture, immediately sterile-filtered ( $0.22 \mu \mathrm{m}$ Millex syringe-driven filter, Millipore) into sterile, $1.5 \mathrm{~mL}$ microcentrifuge tubes, and stored on wet ice until the end of the assay (when all samples had been collected), at which point samples were moved to a refrigerator $\left(4^{\circ} \mathrm{C}\right)$. The presence of unreacted DPTA in the culture filtrate prevented immediate quantification of $\left[\mathrm{NO}_{2}^{-}\right]$ and $\left[\mathrm{NO}_{3}{ }^{-}\right]$, because Griess assays involve a $\mathrm{pH}$ reduction [97], which causes instantaneous release of NO from unreacted DPTA, which would rapidly autoxidize to form $\mathrm{NO}_{2}{ }^{-}, \mathrm{NO}_{3}{ }^{-}$, or escape to the gas phase. To accurately account for contributions of unreacted DPTA to $\mathrm{NO}_{2}{ }^{-}$and $\mathrm{NO}_{3}{ }^{-}$measurements, two steps were taken: (1) samples were stored for at least $24 \mathrm{~h}$ before 
processing, which provided sufficient time for the remaining DPTA to release NO, and for that $\mathrm{NO}$ to form $\mathrm{NO}_{2}{ }^{-}$and $\mathrm{NO}_{3}{ }^{-}$, and (2) a standard curve was generated by adding different concentrations of DPTA $(0,2,5,10,15,20,30$, and $50 \mu \mathrm{M})$ to separate aliquots of growth media $\left(\mathrm{BSM}\right.$ at $37^{\circ} \mathrm{C}$ ) at the beginning of DPTA treatment assays. These standard curve samples were stored under identical conditions $\left(4^{\circ} \mathrm{C}\right)$ for a similar duration as the samples obtained from the DPTA treatment assay, and all samples were assayed for $\mathrm{NO}_{2}{ }^{-}$and $\mathrm{NO}_{3}{ }^{-}$concentrations simultaneously. The DPTA standards yielded a linear calibration curve, relating [DPTA] to $\left[\mathrm{NO}_{2}{ }^{-}\right]$and $\left[\mathrm{NO}_{3}{ }^{-}\right]$(Fig. S9). Based on the DPTA dissociation rate, which was determined from the [NO] curve measured in cell-free media (see Section 4.8.3), the amount of DPTA remaining in the culture at each time point was calculated, and the corresponding concentration of $\mathrm{NO}_{2}{ }^{-}$ and $\mathrm{NO}_{3}{ }^{-}$, as determined from the standard curves, was subtracted from the measured values.

\subsection{Model construction}

Using an existing E. coli K-12 MG1655 model as a template [43], the P. aeruginosa PAO1 kinetic model was constructed following a procedure that was recently employed to generate a quantitative model of NO stress in enterohemorrhagic E. coli O157:H7 [60]. Briefly, all reactions and parameters classified as organism-independent (e.g., glutathione nitrosation,

$\mathrm{N}_{2} \mathrm{O}_{3}$ hydrolysis) remained unchanged, whereas organism-specific components such as enzymes, metabolites, and transcriptional regulatory interactions were added, removed, or changed in accordance with the available body of literature on P. aeruginosa physiology $[25,59,61,64,67,90,98-106]$. We note a difference in the $\mathrm{pH}$ of the growth media used for $P$. aeruginosa compared to that of $E$. coli (7.0 for P. aeruginosa grown in BSM media, 7.4 for $E$. coli grown in MOPS minimal media). Given that the magnitude of this $\mathrm{pH}$ difference was small, 
we assumed that it would only influence the system through its impact on the DPTA NONOate dissociation rate, which was a parameter that was optimized using data from cell-free experiments (Fig. S2).

A BLAST analysis was conducted for each of the enzymes in the original E. coli model to assess the amino acid sequence similarities with the corresponding $P$. aeruginosa homologues, if one existed (Table S9). Although P. aeruginosa possessed homologues for most (16 out of 20) of the enzymes, the amino acid sequences were not highly conserved, with a maximum similarity of $\sim 80 \%$ for the iron-sulfur cluster assembly protein IscU. Given the relatively low sequence similarity among homologous proteins, their associated rate constants were released to be optimized during the initial model training process. The absolute concentrations of metabolites, biomolecules, and enzymes were unavailable for $P$. aeruginosa, and were therefore also released for optimization. Model parameters and species concentrations that were released for optimization were allowed to vary with bounds that were approximated based on literature data available for those components or functions in different bacterial species and/or different experimental conditions (Tables S4-S6).

In addition to releasing parameter values, the model adaptation process also required changes to the network structure (i.e., addition/removal of reactions or changes to their stoichiometry). The $P$. aeruginosa $\mathrm{O}_{2}$-respiration machinery differs greatly from that of E. coli (see Section 2.1.1 and refs [53,61,62]). Therefore, the E. coli cytochrome ubiquinol oxidases were removed and replaced with the five $P$. aeruginosa oxidases (Cyo, CIO, Cbb3-1, Cbb3-2, and Aa3), where ubiquinol served as the electron donor for Cyo and CIO, whereas Cbb3-1, Cbb3-2, and Aa3 received electrons from cytochrome $c$. 
The other network region requiring substantial changes to architecture involved the denitrification reactions and transcriptional regulation of the involved enzymes. Whereas $\mathrm{NO}_{2}{ }^{-}$ and $\mathrm{NO}_{3}{ }^{-}$reductases were omitted from the E. coli model, the $P$. aeruginosa $\mathrm{NO}_{2}{ }^{-}$reductases $\left(\mathrm{NH}_{3}\right.$-forming NirBD and $\mathrm{NO}$-forming $\mathrm{NirS}$ ) and $\mathrm{NO}_{3}{ }^{-}$reductases (NarGHI and NapAB, both $\mathrm{NO}_{2}{ }^{-}$-forming) were incorporated into the present model. Transcriptional regulation of the main NO detoxification enzymes in $P$. aeruginosa (NOD: Fhp and NOR: NorCB) is similar to that of E. coli, in that NOD and NOR are strongly upregulated in the presence of NO. However, while Fhp appears to be regulated independently via the NO-responsive FhpR regulator [25], NorCB regulation is coupled with that of the $\mathrm{NO}_{2}{ }^{-}$and $\mathrm{NO}_{3}{ }^{-}$reductases $[59,61]$. The expression of NorCB, NAR, and NIR are all regulated (directly or indirectly) by the anaerobic response regulator (ANR), which in turn controls expression of the denitrification response regulator (DNR) and a two-component nitrate sensing regulator (NarXL). These three transcription factors (ANR, DNR, and NarXL) are involved (to varying extents) in the regulation of NOR, NAR, and NIR (Fig. S1) [59,61]. To take a mechanistic and physiologically-consistent approach, the ANR, DNR, and NarXL transcriptional regulators were included in the model (see Tables S2 and S3 for reactions and kinetic expressions involving the regulators).

\subsection{Parameter optimization}

\subsubsection{Error minimization}

Model parameters were optimized following a procedure described previously $[43,63]$. Briefly, model simulations were run by numerically integrating the governing system of ordinary differential equations using the Matlab ode $15 s$ stiff ODE solver. The Matlab lsqcurvefit function 
was used to minimize the sum of the squared residuals (SSR) between model simulation data $\left(y_{\text {sim }}\right)$ and experimental measurements $\left(y_{\text {meas }}\right)$, normalized by the experimental variance $\left(\sigma^{2}\right)$ :

$$
S S R^{\prime}=\sum_{i=1}^{n} \frac{\left(y_{i, \text { meas }}-y_{i, \text { sim }}\right)^{2}}{\sigma_{i, \text { meas }}^{2}}
$$

where SSR' represents the variance-normalized SSR. To improve coverage of the solution space, the minimization was repeated for 1,000 iterations, where each iteration began with a different set of randomized initial guess values for the varied parameters, from within their allowed bounds.

\subsubsection{Estimation of parametric uncertainty}

To estimate confidence in the optimized parameter values after the initial SSR' minimization process, and in some cases identify improved optimal solutions, a subsequent Markov chain Monte Carlo (MCMC) procedure was employed [43] using the MCexp function from the HYPERSPACE software package [107]. The SSR' was used as the cost function, and the algorithm was limited to 10,000 parameter evaluations (individual model simulations). Relative quality of fit for each of the parameter sets was quantified by the evidence ratio (ER), which is derived from the Akaike Information Criterion (AIC) [108-110]:

$$
\begin{aligned}
& A I C_{c, i}=n \ln \left(\frac{S S R_{i}^{\prime}}{n}\right)+2 k+\frac{2 k(k+1)}{n-k-1} \\
& \Delta_{i}=A I C_{c, i}-\min \left(A I C_{c}\right)
\end{aligned}
$$




$$
\begin{aligned}
& w_{i}=\frac{\exp \left(-\frac{\Delta_{i}}{2}\right)}{\sum_{i=1}^{P} \exp \left(-\frac{\Delta_{i}}{2}\right)} \\
& E R_{i}=\frac{w_{\text {best }}}{w_{i}}
\end{aligned}
$$

where $n$ is the number of data points, $k$ is the number of fit parameters (plus one, due to the estimation of the SSR [110]), $w_{i}$ is the Akaike weight of parameter set $i, w_{\text {best }}$ is the weight of the best-fit (minimum AIC) parameter set, and $P$ is the total number of parameter sets being compared. Parameter sets with an ER $\geq 10$ (less than $10 \%$ as likely as the best-fit set) were discarded from further analyses $[43,63]$.

\subsubsection{Model training and ensemble generation}

Model parameters were optimized in three different groups or modules: (1) extracellular, (2) respiration, and (3) remaining intracellular and NO detoxification/repair. The extracellular module was optimized first, and included three parameters: $k_{\mathrm{NONOateDPTA}}, k_{\mathrm{NO}-\mathrm{O} 2}$, and $k_{\mathrm{L}} a_{\mathrm{NO}}$, which governed the dissociation rate of DPTA, the rate of NO autoxidation, and the rate of NO exchange with the gas phase, respectively. The volumetric mass transfer coefficient governing $\mathrm{O}_{2}$ exchange with the gas phase, $k_{\mathrm{L}} a_{\mathrm{O} 2}$, was determined independently by measuring dissolved $\left[\mathrm{O}_{2}\right]$ in cell-free growth media following $\mathrm{N}_{2}$-purging, as described previously [36]. The three extracellular parameters were trained on half-hourly measurements of $[\mathrm{NO}],\left[\mathrm{NO}_{2}{ }^{-}\right]$, and $\left[\mathrm{NO}_{3}{ }^{-}\right]$ in cell-free BSM bioreactors treated with $50 \mu \mathrm{M}$ DPTA, over the course of $2 \mathrm{~h}$ (Table S4). After the initial SSR' minimization, the best-fit parameter sets (with ER < 10) were used as initial points in a subsequent MCMC analysis, yielding an ensemble of viable models (all ER < 10) 
differing only in the values of the three extracellular parameters. The confidence interval (CI) of each parameter was calculated as the range (min-max) of that parameter value among the ensemble members.

The respiratory module was optimized second, and included parameters governing concentrations and catalytic activities (e.g., $k_{\text {cat }}, K_{\mathrm{m}}$ ) of the NADH dehydrogenases, respiratory cytochromes, and their associated substrates and products, where a total of 27 parameters were optimized. The parameters were trained on dissolved $\left[\mathrm{O}_{2}\right]$ measurements of bioreactor cultures immediately after inoculation of WT PAO1 cells to an $\mathrm{OD}_{600}$ of 0.05 (in the absence of DPTA treatment) (Table S5). The concentration of $\mathrm{O}_{2}$ was measured continuously, but only for $10 \mathrm{~min}$ post-inoculation, to avoid growth-related effects not captured by the model. Since the three extracellular parameters optimized previously ( $k_{\mathrm{NONOate}, \mathrm{DPTA}}, k_{\mathrm{NO}-\mathrm{O} 2}$, and $\left.k_{\mathrm{L}} a_{\mathrm{NO}}\right)$ have no effect on the system in the absence of NO, they were fixed to their best-fit values during the optimization of the respiratory parameters. A subsequent MCMC analysis was performed with the 27 respiration parameters to generate an ensemble of models, and quantify the corresponding parameter CIs.

After training of the extracellular and respiratory parameters, the remaining intracellular parameters - the majority of which included those related to NO stress, detoxification, and repair-were optimized. A total of 137 parameters were released to be optimized on experimental measurements of $[\mathrm{NO}],\left[\mathrm{O}_{2}\right],\left[\mathrm{NO}_{2}{ }^{-}\right]$, and $\left[\mathrm{NO}_{3}{ }^{-}\right]$in WT PAO1 cultures treated with $50 \mu \mathrm{M}$ DPTA for $30 \mathrm{~min}$ (Table S6). [NO] and [O $\left.\mathrm{O}_{2}\right]$ were measured continuously, whereas $\left[\mathrm{NO}_{2}{ }^{-}\right]$and $\left[\mathrm{NO}_{3}{ }^{-}\right]$were measured immediately after DPTA treatment, and at 30 min posttreatment. In addition to randomly initializing the 137 parameters at the start of each optimization iteration, the previously trained extracellular and respiratory parameters were set to 
values randomly selected from their respective ensembles. In this way, the uncertainties of the extracellular and respiratory parameter values were incorporated into the optimization process, rather than fixing them to single values. Furthermore, in the subsequent MCMC analysis of the 137 intracellular parameters, the extracellular and respiratory parameters were also permitted to vary (within their CIs), to enable a more complete exploration of the viable parameter space (Table S6).

\section{ACKNOWLEDGEMENTS}

We thank Professors Zemer Gitai and Albert Siryaporn for technical assistance with $P$. aeruginosa, and Anna Ren and Ismael Catovic for their initial work on the model. This work was supported by the National Science Foundation (CBET-1453325), and Princeton University (Forese Family Fund for Innovation, Lidow Senior Thesis Fund, start-up funds). 


\section{REFERENCES}

1. Driscoll JA, Brody SL, \& Kollef MH (2007) The epidemiology, pathogenesis and treatment of Pseudomonas aeruginosa infections. Drugs 67(3):351-368.

2. Winstanley C, O'Brien S, \& Brockhurst MA (2016) Pseudomonas aeruginosa Evolutionary Adaptation and Diversification in Cystic Fibrosis Chronic Lung Infections. Trends Microbiol. 24(5):327-337.

3. O'Toole GA \& Kolter R (1998) Flagellar and twitching motility are necessary for Pseudomonas aeruginosa biofilm development. Mol. Microbiol. 30(2):295-304.

4. Drenkard E \& Ausubel FM (2002) Pseudomonas biofilm formation and antibiotic resistance are linked to phenotypic variation. Nature 416(6882):740-743.

5. Breidenstein EBM, de la Fuente-Nunez C, \& Hancock REW (2011) Pseudomonas aeruginosa: all roads lead to resistance. Trends Microbiol. 19(8):419-426.

6. Gellatly SL \& Hancock REW (2013) Pseudomonas aeruginosa: new insights into pathogenesis and host defenses. Pathogens and Disease 67(3):159-173.

7. Poole K (2011) Pseudomonas aeruginosa: resistance to the max. Front Microbiol 2:65.

8. Siryaporn A, Kuchma SL, O'Toole GA, \& Gitai Z (2014) Surface attachment induces Pseudomonas aeruginosa virulence. P Natl Acad Sci USA 111(47):16860-16865.

9. Ketelboeter LM, Potharla VY, \& Bardy SL (2014) NTBC treatment of the pyomelanogenic Pseudomonas aeruginosa clinical isolate PA1111 inhibits pigment production and increases sensitivity to oxidative stress. Curr. Microbiol. 69(3):343-348.

10. Miller LC, et al. (2015) Development of potent inhibitors of pyocyanin production in Pseudomonas aeruginosa. J. Med. Chem. 58(3):1298-1306.

11. Hentzer M, et al. (2003) Attenuation of Pseudomonas aeruginosa virulence by quorum sensing inhibitors. EMBO J. 22(15):3803-3815.

12. Allen RC, Popat R, Diggle SP, \& Brown SP (2014) Targeting virulence: can we make evolution-proof drugs? Nature Reviews Microbiology 12(4):300-308.

13. Cegelski L, Marshall GR, Eldridge GR, \& Hultgren SJ (2008) The biology and future prospects of antivirulence therapies. Nature Reviews Microbiology 6(1):17-27.

14. Escaich S (2008) Antivirulence as a new antibacterial approach for chemotherapy. Curr. Opin. Chem. Biol. 12(4):400-408.

15. Baquero F, Martinez JL, \& Canton R (2008) Antibiotics and antibiotic resistance in water environments. Curr. Opin. Biotechnol. 19(3):260-265.

16. Hauser AR (2009) The type III secretion system of Pseudomonas aeruginosa: infection by injection. Nat. Rev. Microbiol. 7(9):654-665.

17. O'Loughlin CT, et al. (2013) A quorum-sensing inhibitor blocks Pseudomonas aeruginosa virulence and biofilm formation. Proc. Natl. Acad. Sci. U.S.A. 110(44):1798117986.

18. Yoon SS, et al. (2002) Pseudomonas aeruginosa anaerobic respiration in biofilms: Relationships to cystic fibrosis pathogenesis. Dev. Cell 3(4):593-603.

19. Darling KE \& Evans TJ (2003) Effects of nitric oxide on Pseudomonas aeruginosa infection of epithelial cells from a human respiratory cell line derived from a patient with cystic fibrosis. Infect. Immun. 71(5):2341-2349.

20. Kakishima K, Shiratsuchi A, Taoka A, Nakanishi Y, \& Fukumori Y (2007) Participation of nitric oxide reductase in survival of Pseudomonas aeruginosa in LPS-activated macrophages. Biochem. Biophys. Res. Commun. 355(2):587-591. 
21. Bowman LAH, McLean S, Poole RK, \& Fukuto JM (2011) The Diversity of Microbial Responses to Nitric Oxide and Agents of Nitrosative Stress: Close Cousins but Not Identical Twins. Advances in Microbial Physiology, Vol 59 59:135-219.

22. Hyduke DR, Jarboe LR, Tran LM, Chou KJY, \& Liao JC (2007) Integrated network analysis identifies nitric oxide response networks and dihydroxyacid dehydratase as a crucial target in Escherichia coli. P Natl Acad Sci USA 104(20):8484-8489.

23. Poole RK \& Hughes MN (2000) New functions for the ancient globin family: bacterial responses to nitric oxide and nitrosative stress. Mol. Microbiol. 36(4):775-783.

24. Robinson JL, Adolfsen KJ, \& Brynildsen MP (2014) Deciphering nitric oxide stress in bacteria with quantitative modeling. Curr. Opin. Microbiol. 19:16-24.

25. Arai H, Hayashi M, Kuroi A, Ishii M, \& Igarashi Y (2005) Transcriptional regulation of the flavohemoglobin gene for aerobic nitric oxide detoxification by the second nitric oxide-responsive regulator of Pseudomonas aeruginosa. J. Bacteriol. 187(12):3960-3968.

26. Arai H, Igarashi Y, \& Kodama T (1995) The structural genes for nitric oxide reductase from Pseudomonas aeruginosa. Biochim. Biophys. Acta 1261(2):279-284.

27. Chou WK \& Brynildsen MP (2016) A biochemical engineering view of the quest for immune-potentiating anti-infectives. Current Opinion in Chemical Engineering 14:82-92.

28. Barraud N, et al. (2006) Involvement of nitric oxide in biofilm dispersal of Pseudomonas aeruginosa. J. Bacteriol. 188(21):7344-7353.

29. Barraud N, et al. (2009) Nitric oxide signaling in Pseudomonas aeruginosa biofilms mediates phosphodiesterase activity, decreased cyclic di-GMP levels, and enhanced dispersal. J. Bacteriol. 191(23):7333-7342.

30. Van Alst NE, Wellington M, Clark VL, Haidaris CG, \& Iglewski BH (2009) Nitrite Reductase NirS Is Required for Type III Secretion System Expression and Virulence in the Human Monocyte Cell Line THP-1 by Pseudomonas aeruginosa. Infect. Immun. 77(10):4446-4454.

31. Van Alst NE, Picardo KF, Iglewski BH, \& Haidaris CG (2007) Nitrate sensing and metabolism modulate motility, biofilm formation, and virulence in Pseudomonas aeruginosa. Infect. Immun. 75(8):3780-3790.

32. Hassett DJ, et al. (2002) Anaerobic metabolism and quorum sensing by Pseudomonas aeruginosa biofilms in chronically infected cystic fibrosis airways: rethinking antibiotic treatment strategies and drug targets. Adv. Drug Del. Rev. 54(11):1425-1443.

33. Chowdhury A, Zomorrodi AR, \& Maranas CD (2014) k-OptForce: Integrating Kinetics with Flux Balance Analysis for Strain Design. PLoS Comp. Biol. 10(2).

34. Karr JR, et al. (2012) A Whole-Cell Computational Model Predicts Phenotype from Genotype. Cell 150(2):389-401.

35. Khodayari A, Zomorrodi AR, Liao JC, \& Maranas CD (2014) A kinetic model of Escherichia coli core metabolism satisfying multiple sets of mutant flux data. Metab. Eng. 25:50-62.

36. Robinson JL \& Brynildsen MP (2013) A kinetic platform to determine the fate of nitric oxide in Escherichia coli. PLoS Comput Biol 9(5):e1003049.

37. Lancaster JR, Jr. (2006) Nitroxidative, nitrosative, and nitrative stress: kinetic predictions of reactive nitrogen species chemistry under biological conditions. Chem. Res. Toxicol. 19(9):1160-1174.

38. Lim CH, Dedon PC, \& Deen WM (2008) Kinetic analysis of intracellular concentrations of reactive nitrogen species. Chem. Res. Toxicol. 21(11):2134-2147. 
39. Bagci EZ, Vodovotz Y, Billiar TR, Ermentrout B, \& Bahar I (2008) Computational Insights on the Competing Effects of Nitric Oxide in Regulating Apoptosis. Plos One 3(5).

40. Hu TM, Hayton WL, \& Mallery SR (2006) Kinetic modeling of nitric-oxide-associated reaction network. Pharmaceutical Research 23(8):1702-1711.

41. Lewis RS, Tamir S, Tannenbaum SR, \& Deen WM (1995) Kinetic analysis of the fate of nitric oxide synthesized by macrophages in vitro. J. Biol. Chem. 270(49):29350-29355.

42. Robinson JL, Miller RV, \& Brynildsen MP (2014) Model-driven identification of dosing regimens that maximize the antimicrobial activity of nitric oxide. Metabolic Engineering Communications 1:12-18.

43. Robinson JL \& Brynildsen MP (2016) Discovery and dissection of metabolic oscillations in the microaerobic nitric oxide response network of Escherichia coli. Proc Natl Acad Sci USA 113(12):E1757-E1766.

44. Arat S, Bullerjahn GS, \& Laubenbacher R (2015) A network biology approach to denitrification in Pseudomonas aeruginosa. Plos One 10(2):e0118235.

45. Oberhardt MA, Puchałka J, Fryer KE, Martins dos Santos VA, \& Papin JA (2008) Genome-scale metabolic network analysis of the opportunistic pathogen Pseudomonas aeruginosa PAO1. J. Bacteriol. 190(8):2790-2803.

46. Oberhardt MA, Puchalka J, Martins dos Santos VA, \& Papin JA (2011) Reconciliation of genome-scale metabolic reconstructions for comparative systems analysis. PLoS Comput Biol 7(3):e1001116.

47. Biggs MB \& Papin JA (2013) Novel Multiscale Modeling Tool Applied to Pseudomonas aeruginosa Biofilm Formation. Plos One 8(10).

48. Oberhardt MA, Goldberg JB, Hogardt M, \& Papin JA (2010) Metabolic Network Analysis of Pseudomonas aeruginosa during Chronic Cystic Fibrosis Lung Infection. $J$. Bacteriol. 192(20):5534-5548.

49. Varga JJ, et al. (2015) Genotypic and phenotypic analyses of a Pseudomonas aeruginosa chronic bronchiectasis isolate reveal differences from cystic fibrosis and laboratory strains. BMC Genomics 16:883.

50. Kolpen M, et al. (2014) Nitric oxide production by polymorphonuclear leucocytes in infected cystic fibrosis sputum consumes oxygen. Clin. Exp. Immunol. 177(1):310-319.

51. Mason MG, et al. (2009) Cytochrome bd confers nitric oxide resistance to Escherichia coli. Nat. Chem. Biol. 5(2):94-96.

52. Gardner PR, Gardner AM, Martin LA, \& Salzman AL (1998) Nitric oxide dioxygenase: an enzymic function for flavohemoglobin. Proc. Natl. Acad. Sci. U.S.A. 95(18):1037810383.

53. Keseler IM, et al. (2013) EcoCyc: fusing model organism databases with systems biology. Nucleic Acids Res. 41(D1):D605-D612.

54. Cole J (1996) Nitrate reduction to ammonia by enteric bacteria: Redundancy, or a strategy for survival during oxygen starvation? FEMS Microbiol. Lett. 136(1):1-11.

55. Romeo A, et al. (2012) Transcriptional regulation of nitrate assimilation in Pseudomonas aeruginosa occurs via transcriptional antitermination within the nirBD-PA1779-cobA operon. Microbiology-Sgm 158:1543-1552.

56. Winsor GL, et al. (2016) Enhanced annotations and features for comparing thousands of Pseudomonas genomes in the Pseudomonas genome database. Nucleic Acids Res. 44(D1):D646-653. 
57. Kuroki M, Igarashi Y, Ishii M, \& Arai H (2014) Fine-tuned regulation of the dissimilatory nitrite reductase gene by oxygen and nitric oxide in Pseudomonas aeruginosa. Environmental Microbiology Reports 6(6):792-801.

58. Hernandez D \& Rowe JJ (1987) Oxygen regulation of nitrate uptake in denitrifying Pseudomonas aeruginosa. Appl. Environ. Microbiol. 53(4):745-750.

59. Schreiber K, et al. (2007) The anaerobic regulatory network required for Pseudomonas aeruginosa nitrate respiration. J. Bacteriol. 189(11):4310-4314.

60. Robinson JL \& Brynildsen MP (2016) Construction and Experimental Validation of a Quantitative Kinetic Model of Nitric Oxide Stress in Enterohemorrhagic Escherichia coli O157:H7. Bioengineering 3(1).

61. Arai H (2011) Regulation and Function of Versatile Aerobic and Anaerobic Respiratory Metabolism in Pseudomonas aeruginosa. Front Microbiol 2:103.

62. Kanehisa M, Sato Y, Kawashima M, Furumichi M, \& Tanabe M (2016) KEGG as a reference resource for gene and protein annotation. Nucleic Acids Res. 44(D1):D457D462.

63. Adolfsen KJ \& Brynildsen MP (2015) A Kinetic Platform to Determine the Fate of Hydrogen Peroxide in Escherichia coli. PLoS Comp. Biol. 11(11).

64. Zumft WG (1997) Cell biology and molecular basis of denitrification. Microbiol. Mol. Biol. Rev. 61(4):533-616.

65. Gardner PR (2005) Nitric oxide dioxygenase function and mechanism of flavohemoglobin, hemoglobin, myoglobin and their associated reductases. Journal of Inorganic Biochemistry 99(1):247-266.

66. Robinson JL \& Brynildsen MP (2015) An ensemble-guided approach identifies ClpP as a major regulator of transcript levels in nitric oxide-stressed Escherichia coli. Metab. Eng. 31:22-34.

67. Silvestrini MC, Falcinelli S, Ciabatti I, Cutruzzola F, \& Brunori M (1994) Pseudomonas aeruginosa nitrite reductase (or cytochrome oxidase): an overview. Biochimie 76(7):641654.

68. Palmer KL, Brown SA, \& Whiteley M (2007) Membrane-bound nitrate reductase is required for anaerobic growth in cystic fibrosis sputum. J. Bacteriol. 189(12):4449-4455.

69. Van Alst NE, Sherrill LA, Iglewski BH, \& Haidaris CG (2009) Compensatory periplasmic nitrate reductase activity supports anaerobic growth of Pseudomonas aeruginosa PAO1 in the absence of membrane nitrate reductase. Can J Microbiol 55(10):1133-1144.

70. Gardner AM \& Gardner PR (2002) Flavohemoglobin detoxifies nitric oxide in aerobic, but not anaerobic, Escherichia coli - Evidence for a novel inducible anaerobic nitric oxide-scavenging activity. J. Biol. Chem. 277(10):8166-8171.

71. Frey AD \& Kallio PT (2003) Bacterial hemoglobins and flavohemoglobins: versatile proteins and their impact on microbiology and biotechnology. FEMS Microbiol. Rev. 27(4):525-545.

72. Gardner AM, Martin LA, Gardner PR, Dou Y, \& Olson JS (2000) Steady-state and transient kinetics of Escherichia coli nitric-oxide dioxygenase (flavohemoglobin). The B10 tyrosine hydroxyl is essential for dioxygen binding and catalysis. J. Biol. Chem. 275(17):12581-12589.

73. Lyczak JB, Cannon CL, \& Pier GB (2000) Establishment of Pseudomonas aeruginosa infection: lessons from a versatile opportunist. Microbes Infect 2(9):1051-1060. 
74. Govan JR \& Deretic V (1996) Microbial pathogenesis in cystic fibrosis: mucoid Pseudomonas aeruginosa and Burkholderia cepacia. Microbiol Rev 60(3):539-574.

75. Centers for Disease Control and Prevention (CDC) UDoHaHS (2013) Antibiotic resistance threats in the United States, 2013.

76. Rasko DA \& Sperandio V (2010) Anti-virulence strategies to combat bacteria-mediated disease. Nat. Rev. Drug Discov. 9(2):117-128.

77. Deppisch C, et al. (2016) Gaseous nitric oxide to treat antibiotic resistant bacterial and fungal lung infections in patients with cystic fibrosis: a phase I clinical study. Infection.

78. Friedman A, et al. (2011) Susceptibility of Gram-positive and -negative bacteria to novel nitric oxide-releasing nanoparticle technology. Virulence 2(3):217-221.

79. Miller CC, et al. (2013) Inhaled nitric oxide decreases the bacterial load in a rat model of Pseudomonas aeruginosa pneumonia. J Cyst Fibros 12(6):817-820.

80. Yoon SS, et al. (2006) Anaerobic killing of mucoid Pseudomonas aeruginosa by acidified nitrite derivatives under cystic fibrosis airway conditions. J. Clin. Invest. 116(2):436-446.

81. Arai H \& Iiyama K (2013) Role of Nitric Oxide-Detoxifying Enzymes in the Virulence of Pseudomonas aeruginosa against the Silkworm, Bombyx mori. Bioscience

Biotechnology and Biochemistry 77(1):198-200.

82. Platzen L, Koch-Koerfges A, Weil B, Brocker M, \& Bott M (2014) Role of flavohaemoprotein Hmp and nitrate reductase NarGHJI of Corynebacterium glutamicum for coping with nitrite and nitrosative stress. FEMS Microbiol. Lett. 350(2):239-248.

83. Sasaki Y, et al. (2016) Nitrogen oxide cycle regulates nitric oxide levels and bacterial cell signaling. Sci Rep 6:22038.

84. Igamberdiev AU, Bykova NV, Shah JK, \& Hill RD (2010) Anoxic nitric oxide cycling in plants: participating reactions and possible mechanisms. Physiol. Plant. 138(4):393-404.

85. Reutov VP \& Sorokina EG (1998) NO-synthase and nitrite-reductase components of nitric oxide cycle. Biochemistry (Mosc) 63(7):874-884.

86. Bogdan C (2001) Nitric oxide and the immune response. Nat. Immunol. 2(10):907-916.

87. Nathan C (1992) Nitric-Oxide as a Secretory Product of Mammalian-Cells. FASEB J. 6(12):3051-3064.

88. Pacher P, Beckman JS, \& Liaudet L (2007) Nitric oxide and peroxynitrite in health and disease. Physiol. Rev. 87(1):315-424.

89. Berks BC, Ferguson SJ, Moir JWB, \& Richardson DJ (1995) Enzymes and associated electron transport systems that catalyse the respiratory reduction of nitrogen oxides and oxyanions. Biochimica Et Biophysica Acta-Bioenergetics 1232(3):97-173.

90. Silvestrini MC, Tordi MG, Musci G, \& Brunori M (1990) The reaction of Pseudomonas nitrite reductase and nitrite. A stopped-flow and EPR study. J. Biol. Chem. 265(20):11783-11787.

91. Amato SM \& Brynildsen MP (2014) Nutrient transitions are a source of persisters in Escherichia coli biofilms. Plos One 9(3):e93110.

92. Amato SM, Orman MA, \& Brynildsen MP (2013) Metabolic control of persister formation in Escherichia coli. Mol. Cell 50(4):475-487.

93. Lesic B \& Rahme LG (2008) Use of the lambda Red recombinase system to rapidly generate mutants in Pseudomonas aeruginosa. BMC Mol. Biol. 9.

94. Shen Y, Siryaporn A, Lecuyer S, Gitai Z, \& Stone HA (2012) Flow directs surfaceattached bacteria to twitch upstream. Biophys. J. 103(1):146-151. 
95. Kuwayama H, et al. (2002) PCR-mediated generation of a gene disruption construct without the use of DNA ligase and plasmid vectors. Nucleic Acids Res. 30(2).

96. Wolff JA, MacGregor CH, Eisenberg RC, \& Phibbs PV, Jr. (1991) Isolation and characterization of catabolite repression control mutants of Pseudomonas aeruginosa PAO. J. Bacteriol. 173(15):4700-4706.

97. Tsikas D (2007) Analysis of nitrite and nitrate in biological fluids by assays based on the Griess reaction: appraisal of the Griess reaction in the L-arginine/nitric oxide area of research. J Chromatogr B Analyt Technol Biomed Life Sci 851(1-2):51-70.

98. Arai H, et al. (2014) Enzymatic Characterization and In Vivo Function of Five Terminal Oxidases in Pseudomonas aeruginosa. J. Bacteriol. 196(24):4206-4215.

99. Carlson CA, Ferguson LP, \& Ingraham JL (1982) Properties of dissimilatory nitrate reductase purified from the denitrifier Pseudomonas aeruginosa. J. Bacteriol. 151(1):162171.

100. Cutruzzola F, Arese M, Grasso S, Bellelli A, \& Brunori M (1997) Mutagenesis of nitrite reductase from Pseudomonas aeruginosa: tyrosine-10 in the $\mathrm{c}$ heme domain is not involved in catalysis. FEBS Lett. 412(2):365-369.

101. Cutruzzola F, et al. (2001) The nitrite reductase from Pseudomonas aeruginosa: Essential role of two active-site histidines in the catalytic and structural properties. P Natl Acad Sci USA 98(5):2232-2237.

102. Frey AD, Farres J, Bollinger CJ, \& Kallio PT (2002) Bacterial hemoglobins and flavohemoglobins for alleviation of nitrosative stress in Escherichia coli. Appl. Environ. Microbiol. 68(10):4835-4840.

103. Fukumori Y, Nakayama K, \& Yamanaka T (1985) Cytochrome c oxidase of Pseudomonas AM 1: purification, and molecular and enzymatic properties. $J$ Biochem 98(2):493-499.

104. SooHoo CK \& Hollocher TC (1991) Purification and characterization of nitrous oxide reductase from Pseudomonas aeruginosa strain P2. J. Biol. Chem. 266(4):2203-2209.

105. Shapleigh JP, Davies KJP, \& Payne WJ (1987) Detergent Inhibition of Nitric-Oxide Reductase-Activity. Biochimica Et Biophysica Acta 911(3):334-340.

106. Kawakami T, Kuroki M, Ishii M, Igarashi Y, \& Arai H (2010) Differential expression of multiple terminal oxidases for aerobic respiration in Pseudomonas aeruginosa. Environ. Microbiol. 12(6):1399-1412.

107. Zamora-Sillero E, Hafner M, Ibig A, Stelling J, \& Wagner A (2011) Efficient characterization of high-dimensional parameter spaces for systems biology. Bmc Systems Biology 5.

108. Akaike H (1973) Information theory and extension of the maximum likelihood principle. Second International Symposium on Information Theory, eds Petrov BN \& Csaki F (Akadémiai Kiadó, Budapest), pp 267-281.

109. Hurvich CM \& Tsai CL (1989) Regression and Time-Series Model Selection in Small Samples. Biometrika 76(2):297-307.

110. Turkheimer FE, Hinz R, \& Cunningham VJ (2003) On the undecidability among kinetic models: From model selection to model averaging. Journal of Cerebral Blood Flow and Metabolism 23(4):490-498. 